TRANSACTIONS OF THE

AMERICAN MATHEMATICAL SOCIETY

Volume 357, Number 8, Pages 3179-3199

S 0002-9947(04)03643-8

Article electronically published on September 2, 2004

\title{
SIGNATURE OF RELATIONS IN MAPPING CLASS GROUPS AND NON-HOLOMORPHIC LEFSCHETZ FIBRATIONS
}

\author{
HISAAKI ENDO AND SEIJI NAGAMI
}

Dedicated to Professor Yukio Matsumoto for his 60th birthday

\begin{abstract}
We introduce the notion of signature for relations in mapping class groups and show that the signature of a Lefschetz fibration over the 2-sphere is the sum of the signatures for basic relations contained in its monodromy. Combining explicit calculations of the signature cocycle with a technique of substituting positive relations, we give some new examples of non-holomorphic Lefschetz fibrations of genus 3,4 and 5 which violate slope bounds for nonhyperelliptic fibrations on algebraic surfaces of general type.
\end{abstract}

\section{INTRODUCTION}

The study of Lefschetz fibrations has turned out to be interesting and important by virtue of the remarkable works of Donaldson [10] and Gompf [17] which demonstrate a close relationship between symplectic 4-manifolds and Lefschetz fibrations.

The geography problem of Lefschetz fibrations is one of the most interesting topics to be investigated. Originally, the geography problem for complex surfaces was to find minimal surfaces of general type with a prescribed pair of Chern numbers. Using the pair of Euler characteristic and signature instead of the pair of Chern numbers, we can consider also the geography problem of Lefschetz fibrations. The Euler characteristic of a given Lefschetz fibration is easily computed, while the signature is not. If the Lefschetz fibration is over the 2-sphere, Ozbagci [33] and Smith [36] gave signature formulae: the former's is suited for explicit computation and the latter's for showing some qualitative properties of Lefschetz fibrations. If the Lefschetz fibration is hyperelliptic, a local signature, which is a generalization of the $\sigma$-number [28] and the fractional signature [29] due to Matsumoto, was defined by the first author [12] and a method of computing signatures was established.

It is also interesting to find various kinds of examples of non-holomorphic Lefschetz fibrations, which imply the difference between the geography of algebraic surfaces fibered over curves and that of Lefschetz fibrations. Ozbagci and Stipsicz 34] constructed examples of non-holomorphic genus-2 Lefschetz fibrations whose total space never appear in the table of the Enriques-Kodaira classification of complex surfaces. Korkmaz [25] generalized their examples to higher genera. Fintushel

Received by the editors November 16, 2003.

2000 Mathematics Subject Classification. Primary 57R20; Secondary 57N13, 20F05, 14D06.

Key words and phrases. Signature, mapping class group, Lefschetz fibration, relation, signature cocycle, slope. 
and Stern [14] produced minimal symplectic Lefschetz fibrations which do not satisfy the Noether inequality.

In the present paper we would like to propose a useful method of computing signatures of Lefschetz fibrations and give some new examples of non-holomorphic Lefschetz fibrations. We first introduce the notion of signature for relations in mapping class groups. The signature of a Lefschetz fibration over the 2 -sphere is equal to the sum of the signatures for basic relations appearing in its monodromy. Combining explicit computations of signatures for basic relations with a technique of substituting positive relations, which is a generalization of a method of Fuller and Smith [37, we construct new examples of non-holomorphic Lefschetz fibrations of genus 3,4 and 5 which violate lower bounds of the slope of non-hyperelliptic fibrations in algebraic geometry.

This paper is organized as follows. In Section 2 we recall a well-known theorem of Hopf and some facts on 2-cocycles of groups. In Section 3 we define the signature of a relator of mapping class groups and carry out explicit computations of signatures for basic relators. In Section 4 we construct examples of non-holomorphic Lefschetz fibrations using the results of Section 3 and some results on slope bounds for algebraic surfaces.

The idea of "the signature of a relation" occurred to the first author when he worked on signature of surface bundles at the University of Munich in 2000 with the co-authors of [13. He is grateful to the co-authors of [13], especially D. Kotschick for helpful discussions and comments. The authors are grateful to K. Konno for a detailed explanation of his works on the lower bound of the slope of non-hyperelliptic fibrations and to T. Morifuji for useful comments on his formula of Meyer's function in [30].

\section{Hopf's TheOREM AND 2-COCYClE}

In this section we review some facts about cohomology of groups (cf. Brown [6] and Meyer [27]).

Let $G$ be a group. We define the homology $H_{*}(G)$ of $G$ as the homology of the co-invariants of the standard resolution of $\mathbb{Z}$ over $\mathbb{Z} G$. The standard chain complex of $G$ is denoted by $C_{*}(G)$. We describe the second homology group $H_{2}(G)$ in terms of a given presentation of $G$.

Theorem 2.1 (Hopf [19]). Let $G$ be a group, $F$ a free group and $\pi: F \rightarrow G$ an epimorphism. Then the following isomorphism holds:

$$
H_{2}(G) \cong R \cap[F, F] /[R, F],
$$

where $R=\operatorname{Ker} \pi$ is the kernel of $\pi$.

An explicit homomorphism which induces the isomorphism in Hopf's theorem is given as follows.

Lemma 2.2. Let $G$ be a group, $F=F(S)$ the free group generated by a set $S$ and $\pi: F \rightarrow G$ an epimorphism. The homomorphism

$$
R \rightarrow C_{2}(G): r \mapsto \sum_{s \in S}\left[\overline{\frac{\partial r}{\partial s}} \mid \bar{s}\right]
$$

induces the isomorphism

$$
R \cap[F, F] /[R, F] \rightarrow H_{2}(G)=H_{2}\left(C_{*}(G)\right),
$$


where $\partial / \partial s: \mathbb{Z} F \rightarrow \mathbb{Z} F$ is the Fox free derivative with respect to $s, \bar{f}$ is the image of $f \in F$ under $\pi$ and the symbol $[\cdot \mid \cdot]$ stands for the bar notation extended linearly over $\mathbb{Z} G$.

Proof. See Brown [6], §2.5, Exercise 4.

Using the lemma above, we have the following description of the evaluation map $H_{2}(G) \rightarrow \mathbb{Z}$ for the cohomology class of a 2-cocycle of $G$.

Proposition 2.3 (cf. Meyer [27], §3). Let $G$ be a group, $F=F(S)$ the free group generated by a set $S$ and $\pi: F \rightarrow G$ an epimorphism. If a 2-cocycle $z: G \times G \rightarrow \mathbb{Z}$ of $G$ satisfies the condition

$$
z(g, 1)=z(1, g)=z\left(g, g^{-1}\right)=0 \quad \text { for all } g \in G
$$

then the homomorphism $H_{2}\left(C_{*}(G)\right) \rightarrow \mathbb{Z}$ defined by the cohomology class of $z$ together with the Kronecker product is induced by the homomorphism

$$
\begin{gathered}
c: R \longrightarrow \mathbb{Z}: r \mapsto \sum_{j=1}^{m} z\left(\overline{s_{1} \cdots s_{j-1}}, \overline{s_{j}}\right) \\
\left(r=s_{1} \cdots s_{m}, s_{1}, \ldots, s_{m} \in S \cup S^{-1}\right)
\end{gathered}
$$

under the isomorphism in Lemma 2.2 .

Proof. Let $r$ be an element of $R$. We express $r$ as a word in $s_{1}, \ldots, s_{m}: r=$ $s_{1}^{\epsilon_{1}} \cdots s_{m}^{\epsilon_{m}}$, where $\epsilon_{1}, \ldots, \epsilon_{m} \in\{ \pm 1\}$. By virtue of Lemma 2.2, the image of $r$ under the composition $c: R \rightarrow C_{2}(G) \rightarrow \mathbb{Z}$ is equal to

$$
\sum_{s \in S} z\left(\overline{\frac{\partial r}{\partial s}}, \bar{s}\right)=\sum_{j=1}^{m} z\left(\overline{\frac{\partial r}{\partial s_{j}}}, \overline{s_{j}}\right)=\sum_{j=1}^{m} z\left(\overline{s_{1}^{\epsilon_{1}} \cdots s_{j-1}^{\epsilon_{j-1}} \frac{\partial s_{j}^{\epsilon_{j}}}{\partial s_{j}}}, \overline{s_{j}}\right) .
$$

If $\epsilon_{j}=+1$, we have

$$
\begin{aligned}
z\left(\overline{s_{1}^{\epsilon_{1}} \cdots s_{j-1}^{\epsilon_{j-1}} \frac{\partial s_{j}^{\epsilon_{j}}}{\partial s_{j}}}, \overline{s_{j}}\right) & =z\left(\overline{s_{1}^{\epsilon_{1}} \cdots s_{j-1}^{\epsilon_{j-1}} \frac{\partial s_{j}}{\partial s_{j}}}, \overline{s_{j}}\right)=z\left(\overline{s_{1}^{\epsilon_{1}} \cdots s_{j-1}^{\epsilon_{j-1}}}, \overline{s_{j}}\right) \\
& =z\left(\overline{s_{1}^{\epsilon_{1}} \cdots s_{j-1}^{\epsilon_{j-1}}}, \overline{s_{j}^{\epsilon_{j}}}\right)
\end{aligned}
$$

and if $\epsilon_{j}=-1$, we have

$$
\begin{aligned}
z\left(\overline{s_{1}^{\epsilon_{1}} \cdots s_{j-1}^{\epsilon_{j-1}} \frac{\partial s_{j}^{\epsilon_{j}}}{\partial s_{j}}}, \overline{s_{j}}\right) & =z\left(\overline{s_{1}^{\epsilon_{1}} \cdots s_{j-1}^{\epsilon_{j-1}} \frac{\partial s_{j}^{-1}}{\partial s_{j}}}, \overline{s_{j}}\right)=z\left(\overline{s_{1}^{\epsilon_{1}} \cdots s_{j-1}^{\epsilon_{j-1}} \cdot s_{j}^{-1}}, \overline{s_{j}}\right) \\
& =z\left(\overline{s_{1}^{\epsilon_{1}} \cdots s_{j-1}^{\epsilon_{j-1}}}, \overline{s_{j}^{-1}}\right)-z\left(\overline{s_{1}^{\epsilon_{1}} \cdots s_{j-1}^{\epsilon_{j-1}}}, \overline{1}\right)-z\left(\overline{s_{j}^{-1}}, \overline{s_{j}}\right) \\
& =z\left(\overline{s_{1}^{\epsilon_{1}} \cdots s_{j-1}^{\epsilon_{j-1}}}, \overline{s_{j}^{\epsilon_{j}}}\right)
\end{aligned}
$$

from $(*)$ and the cocycle condition.

Remark 2.4. The homomorphism $c: R \rightarrow \mathbb{Z}$ naturally extend to a map $c: F \rightarrow \mathbb{Z}$ by virtue of the definition above. It satisfies $c(x y)=c(x)+c(y)+z(\bar{x}, \bar{y})$ for $x, y \in F$ (i.e. $\delta c=-\pi^{*} z$ ) (see the formula (25) of Meyer [27]). 


\section{Signature of RElations in MAPPing ClASS GROUPS}

In this section we introduce the notion of a signature for relations in mapping class groups of surfaces and calculate it for the relations in presentations due to Gervais [16] and Luo [26].

Let $\Sigma_{g}$ be a closed oriented surface of genus $g$ and $\mathcal{M}_{g}$ the mapping class group of $\Sigma_{g}$, namely the group of all isotopy classes of orientation-preserving diffeomorphisms of $\Sigma_{g}$. We follow the functional notation: for $\varphi, \psi \in \mathcal{M}_{g}$, the symbol $\varphi \psi$ means that we apply $\psi$ first and then $\varphi$. We denote by $\mathcal{F}$ the free group generated by all isotopy classes $\mathcal{S}$ of simple closed curves on $\Sigma_{g}: \mathcal{F}=F(\mathcal{S})$. There is a natural homomorphism $\varpi: \mathcal{F} \rightarrow \mathcal{M}_{g}$ which sends (the isotopy class of) a simple closed curve $a$ on $\Sigma_{g}$ to the right-handed Dehn twist $t_{a}$ along $a$. We often denote the image $\varpi(W)$ of a word $W$ in generators $\mathcal{S}$ by $\bar{W}$. In particular, $\varpi(a)=t_{a}=\bar{a}$ for a simple closed curve $a \in \mathcal{S}$. It is well known as a theorem of Dehn [9] that this homomorphism $\varpi$ is surjective. We set $\mathcal{R}:=\operatorname{Ker} \varpi$ and call each element of $\mathcal{R}$ a relator in the generators $\mathcal{S}$ of $\mathcal{M}_{g}$.

Gervais [16] gave an infinite presentation of $\mathcal{M}_{g}$ with generators $\mathcal{S}$.

Theorem 3.1 (Gervais [16]). The kernel $\mathcal{R}$ of $\varpi$ is normally generated by the following relators:

(I) all the braid relators $T_{0}, T_{1}$ and $T_{2_{0}}$;

(II) all the chain relators $C_{3}$ of length 3 ;

(III) all the lantern relators $L$.

If $g \geq 1$, (II) and (III) can be replaced by

(IV) all the star relators $E$.

Luo [26] improved Gervais' infinite presentation to show that all the relators were essentially discovered by Dehn.

Theorem 3.2 (Luo [26]). The kernel $\mathcal{R}$ of $\varpi$ is normally generated by the following relators:

(I) all the braid relators $T_{0}$ and $T_{1}$;

(II) all the chain relators $C_{2}$ of length 2 ;

(III) all the lantern relators $L$.

We will give definitions of the relators exhibited in the two theorems above together with their signature computations in the latter half of this section.

We now define the signature of a relator of $\mathcal{M}_{g}$.

Definition 3.3. We set $G=\mathcal{M}_{g}, F=\mathcal{F}, R=\mathcal{R}, \pi=\varpi$ and $z=\tau_{g}$ in Proposition 2.3, where $\tau_{g}: \mathcal{M}_{g} \times \mathcal{M}_{g} \rightarrow \mathbb{Z}$ is the signature cocycle due to Meyer [27] (see Appendix A). Then we get an explicit homomorphism $c_{g}: \mathcal{R} \rightarrow \mathbb{Z}$ inducing the evaluation $H_{2}(G) \rightarrow \mathbb{Z}$. For a relator $\varrho \in \mathcal{R}$, we define $I_{g}(\varrho):=-c_{g}(\varrho)-s(\varrho)$ and call it the signature of $\varrho$, where $s(\varrho)$ is the total exponent of separating simple closed curves contained in the word $\varrho$. For example, the signature $I_{g}(A)$ of the identity relator $A:=a \in \mathcal{R}$, where $a$ is a null-homotopic simple closed curve on $\Sigma_{g}$, is equal to -1 .

A compact connected oriented surface of genus $g$ with $n$ boundary components is denoted by $\Sigma_{g, n}$. For (isotopy classes of) simple closed curves $a, b$, we denote their geometric intersection number by $i(a, b)$.

Let $\varrho, \varrho^{\prime} \in \mathcal{R}$ be relators. We express $\varrho$ as a word in generators $\mathcal{S}: \varrho=c_{1}^{\epsilon_{1}} \cdots c_{m}^{\epsilon_{m}}$, where $c_{1}, \ldots, c_{m}$ are simple closed curves on $\Sigma_{g}$ and $\epsilon_{1}, \ldots, \epsilon_{m}= \pm 1$. If there 
exists an orientation-preserving homeomorphism $h: \Sigma_{g} \rightarrow \Sigma_{g}$ such that $\varrho^{\prime}=$ $h\left(c_{1}\right)^{\epsilon_{1}} \cdots h\left(c_{m}\right)^{\epsilon_{m}}$, we call that $\varrho$ and $\varrho^{\prime}$ are topologically equivalent or have the same topological type. We call $\varrho$ a positive relator if $\epsilon_{1}=\cdots=\epsilon_{m}=+1$.

Definition 3.4 (Smith [37]). Let $\varrho \in \mathcal{R}$ be a relator and $\varsigma \in \mathcal{R}$ a (positive) relator. Suppose that there exist (positive) words $U, V, W_{1}, W_{2} \in \mathcal{F}$ such that $\varrho=W_{1}^{-1} W_{2}$ and $\varsigma=U W_{1} V$. Then we can construct a new (positive) relator $\varsigma^{\prime}=\varsigma V^{-1} \varrho V=U W_{2} V$ in $\mathcal{R}$. This operation is called a $\varrho$-substitution to $\varsigma$. When $\varsigma^{\prime}$ is obtained by applying a sequence of $\varrho^{ \pm 1}$-substitutions to $\varsigma$, we denote it by $\varsigma \equiv \varsigma^{\prime}(\bmod \varrho)$.

Lemma 3.5. The signature $I_{g}$ for relators of $\mathcal{M}_{g}$ has the following properties:

(1) $I_{g}\left(\varrho^{-1}\right)=-I_{g}(\varrho)$ for $\varrho \in \mathcal{R}$;

(2) $I_{g}\left(W \varrho W^{-1}\right)=I_{g}(\varrho)$ for $\varrho \in \mathcal{R}$ and $W \in \mathcal{F}$;

(3) $I_{g}\left(\varrho_{1} \varrho_{2}\right)=I_{g}\left(\varrho_{1}\right)+I_{g}\left(\varrho_{2}\right)$ for $\varrho_{1}, \varrho_{2} \in \mathcal{R}$;

(4) $I_{g}\left(\varsigma^{\prime}\right)=I_{g}(\varsigma)+I_{g}(\varrho)$ if $\varsigma^{\prime}$ is obtained by applying a $\varrho$-substitution to $\varsigma$, where $\varrho, \varsigma, \varsigma^{\prime} \in \mathcal{R}$;

(5) $I_{g}(\varrho)=I_{g}\left(\varrho^{\prime}\right)$ if $\varrho \in \mathcal{R}$ and $\varrho^{\prime} \in \mathcal{R}$ are topologically equivalent.

Proof. (1)-(3): It is clear that $s\left(\varrho^{-1}\right)=-s(\varrho), s\left(W \varrho W^{-1}\right)=s(\varrho)$ and $s\left(\varrho_{1} \varrho_{2}\right)=$ $s\left(\varrho_{1}\right)+s\left(\varrho_{2}\right)$ for $\varrho, \varrho_{1}, \varrho_{2} \in \mathcal{R}$ and $W \in \mathcal{F}$. It is trivial that $c_{g}\left(\varrho^{-1}\right)=-c_{g}(\varrho)$ and $c_{g}\left(\varrho_{1} \varrho_{2}\right)=c_{g}\left(\varrho_{1}\right)+c_{g}\left(\varrho_{2}\right)$ for $\varrho, \varrho_{1}, \varrho_{2} \in \mathcal{R}$ because $c_{g}$ is a homomorphism on $\mathcal{R}$. A proof of $c_{g}\left(W \varrho W^{-1}\right)=c_{g}(\varrho)$ for $\varrho \in \mathcal{R}$ and $W \in \mathcal{F}$ are found in Meyer [27], p. 253. (4): We use the notation of Definition 3.4 above. Then we have

$$
I_{g}\left(\varsigma^{\prime}\right)=I_{g}(\varsigma)+I_{g}\left(V^{-1} \varrho V\right)=I_{g}(\varsigma)+I_{g}(\varrho)
$$

from (2) and (3). We postpone the proof of (5) until the next subsection.

3.1. Braid relation. Let $a, b$ be simple closed curves on $\Sigma_{g}$ and put $c=t_{b}(a)$. The relation

$$
t_{c}=t_{b} t_{a} t_{b}^{-1}
$$

in $\mathcal{M}_{g}$ is called the braid relation. If $i(a, b)=n$, we put $T_{n}=T(a, b):=b a b^{-1} c^{-1} \in$ $\mathcal{R}$. Especially, we denote $T_{2}$ by $T_{2_{0}}$ if the algebraic intersection number of $a$ and $b$ is equal to 0 .

Proposition 3.6. The signature $I_{g}\left(T_{n}\right)$ of a braid relator $T_{n}(n=0,1,2, \ldots)$ is equal to 0.

Proof. We first note that $c_{g}: \mathcal{R} \rightarrow \mathbb{Z}$ can naturally be extended to $c_{g}: \mathcal{F} \rightarrow \mathbb{Z}$ and satisfies $c_{g}(u v)=c_{g}(u)+c_{g}(v)+\tau_{g}(\bar{u}, \bar{v})$ for $u, v \in \mathcal{F}$ (see Remark 2.4). Then we have

$$
\begin{aligned}
c_{g}\left(T_{n}\right) & =c_{g}\left(b a b^{-1} c^{-1}\right)=c_{g}\left(b a b^{-1}\right)+c_{g}\left(c^{-1}\right)+\tau_{g}\left(\overline{b a b^{-1}}, \overline{c^{-1}}\right) \\
& =c_{g}(a)-c_{g}(c)+\tau_{g}\left(\bar{c}, \overline{c^{-1}}\right) \\
& =0-0+0=0
\end{aligned}
$$

(cf. Meyer 27], p. 253). Hence we have $I_{g}\left(T_{n}\right)=-c_{g}\left(T_{n}\right)-s\left(T_{n}\right)=-0-0=0$.

Proof of Lemma 3.5 (5). We express $\varrho$ as a word in generators $\mathcal{S}: \varrho=c_{1}^{\epsilon_{1}} \cdots c_{m}^{\epsilon_{m}}$, where $c_{1}, \ldots, c_{m}$ are simple closed curves on $\Sigma_{g}$ and $\epsilon_{1}, \ldots, \epsilon_{m}= \pm 1$. There exists an orientation-preserving homeomorphism $h: \Sigma_{g} \rightarrow \Sigma_{g}$ such that $\varrho^{\prime}=$ 
$h\left(c_{1}\right)^{\epsilon_{1}} \cdots h\left(c_{m}\right)^{\epsilon_{m}}$. Choose a word $W \in \mathcal{F}$ such that $\varpi(W)=h$. Applying $T_{n}^{-1}$ substitutions to each $h\left(c_{i}\right)$, we have $h\left(c_{i}\right) \equiv W c_{i} W^{-1}\left(\bmod T_{n}\right)$. Hence we get

$$
I_{g}\left(\varrho^{\prime}\right)=I_{g}\left(h\left(c_{1}\right)^{\epsilon_{1}} \cdots h\left(c_{m}\right)^{\epsilon_{m}}\right)=I_{g}\left(W c_{1}^{\epsilon_{1}} \cdots c_{m}^{\epsilon_{m}} W^{-1}\right)=I_{g}(\varrho)
$$

from (2), (4) and Proposition 3.6.

Remark 3.7. (1) For each $n=0,1,2, \ldots$, the topological type of $T_{n}$ need not be unique. $T_{1}$ has a unique topological type because both $a$ and $b$ must be nonseparating.

(2) If $i(a, b)=1$, we also have the braid relation $t_{b}=t_{a} t_{c} t_{a}^{-1}$. This relation together with the original relation $t_{c}=t_{b} t_{a} t_{b}^{-1}$ yields Artin's relation $t_{b}=$ $t_{a} t_{b} t_{a} t_{b}^{-1} t_{a}^{-1}$. If we put $T_{A}:=a b a b^{-1} a^{-1} b^{-1}$, then we have $I_{g}\left(T_{A}\right)=-c_{g}\left(T_{A}\right)=$ $-2 c_{g}\left(T_{1}\right)=0$.

\subsection{Chain relation.}

Definition 3.8 (cf. Johnson [21], Wajnryb [44]). An ordered $n$-tuple $\left(c_{1}, \ldots, c_{n}\right)$ of simple closed curves on $\Sigma_{g}$ is called a chain of length $n$ if it satisfies the next conditions:

(i) $c_{i}$ and $c_{i+1}$ intersect transversely at one point $(i=1, \ldots, n-1)$;

(ii) $c_{i} \cap c_{j}=\emptyset$ if $|i-j|>1$.

When the length $n$ is even, a regular neighborhood of a chain $\left(c_{1}, \ldots, c_{n}\right)$ is a subsurface of $\Sigma_{g}$ which is of genus $h=n / 2$ and has one boundary component. We denote a simple closed curve parallel to the boundary by $d$. The relation

$$
t_{d}=\left(t_{c_{1}} \cdots t_{c_{2 h}}\right)^{4 h+2}
$$

is called the chain relation of length $2 h$, or the even chain relation (see Wajnryb [44]). We put $C_{2 h}:=C\left(c_{1}, \ldots, c_{2 h}\right)=\left(c_{1} \cdots c_{2 h}\right)^{4 h+2} d^{-1} \in \mathcal{R}$. The topological type of $C_{2 h}$ is unique.

Proposition 3.9. The signature $I_{g}\left(C_{2 h}\right)$ of a chain relator $C_{2 h}$ of length $2 h$ is equal to $-4 h(h+1)+1$.

Proof. From direct computations of the signature cocycle, we have

$$
\begin{aligned}
c_{g}\left(C_{2 h}\right) & =c_{g}\left(\left(c_{1} \cdots c_{2 h}\right)^{4 h+2}\right)-c(d)+\tau_{g}\left(\bar{d}, \overline{d^{-1}}\right) \\
& =(2 h+1) c_{g}\left(\left(c_{1} \cdots c_{2 h}\right)^{2}\right)+\sum_{i=1}^{2 h-1} \tau_{g}\left(\overline{\left(c_{1} \cdots c_{2 h}\right)^{2}}, \overline{\left(c_{1} \cdots c_{2 h}\right)^{2 i}}\right) \\
& =(2 h+1)\left(2 c_{g}\left(c_{1} \cdots c_{2 h}\right)+\tau_{g}\left(\overline{c_{1} \cdots c_{2 h}}, \overline{c_{1} \cdots c_{2 h}}\right)\right)+2 h \\
& =(2 h+1)(0+2 h)+2 h \\
& =4 h(h+1) .
\end{aligned}
$$

See also [12] Lemma 3.5 for similar computations. This completes the proof because $s\left(C_{2 h}\right)=-1$.

When the length $n$ is odd, a regular neighborhood of a chain $\left(c_{1}, \ldots, c_{n}\right)$ is a subsurface of $\Sigma_{g}$ which is of genus $h=(n-1) / 2$ and has two boundary components. We denote simple closed curves parallel to two boundary components by $d_{1}$ and $d_{2}$. The relation

$$
t_{d_{1}} t_{d_{2}}=\left(t_{c_{1}} \cdots t_{c_{2 h+1}}\right)^{2 h+2}
$$


is called the chain relation of length $2 h+1$, or the odd chain relation (see Wajnryb 44]). We put $C_{2 h+1}:=C\left(c_{1}, \ldots, c_{2 h+1}\right)=\left(c_{1} \cdots c_{2 h+1}\right)^{2 h+2} d_{1}^{-1} d_{2}^{-1} \in \mathcal{R}$. The topological type of $C_{2 h+1}$ is not unique. We denote $C_{2 h+1}$ also by $C_{2 h+1, \mathrm{I}}$ if both $d_{1}$ and $d_{2}$ are non-separating, while we denote it by $C_{2 h+1, \mathrm{II}}$ if both $d_{1}$ and $d_{2}$ are separating.

Proposition 3.10. The signature $I_{g}\left(C_{2 h+1}\right)$ of a chain relator $C_{2 h+1}$ of length $2 h+1$ is equal to $-2 h(h+2)$. In particular, it does not depend on the topological type of $C_{2 h+1}$.

Proof. If $d_{1}$ and $d_{2}$ are separating, then we have

$$
\begin{aligned}
I_{g}\left(C_{2 h+1, \mathrm{II}}\right) & =-c_{g}\left(C_{2 h+1, \mathrm{II}}\right)-s\left(C_{2 h+1, \mathrm{II}}\right)=-2(h+1)^{2}-(-2) \\
& =-2 h(h+2)
\end{aligned}
$$

from the formula (30) of Meyer [27.

If $d_{1}$ and $d_{2}$ are non-separating, we can compute the value with the help of a work by Arakawa and Ashikaga 22 or by Morifuji [30] as follows. Choose a hyperelliptic involution $\iota: \Sigma_{g} \rightarrow \Sigma_{g}$ so that the chain $\left(c_{1}, \ldots, c_{2 h+1}\right)$ is invariant under the action of $\iota$. Then the map $t_{d_{1}} t_{d_{2}}=\left(t_{c_{1}} \cdots t_{c_{2 h+1}}\right)^{2 h+2}$ is the monodromy of a degeneration of hyperelliptic curves, whose local signature is equal to $(2 h(g-h-1)-1) /(2 g+1)-1$ (see [2]). According to a theorem of Terasoma [42], the value $\phi_{g}\left(t_{d_{1}} t_{d_{2}}\right)$ of Meyer's function on $t_{d_{1}} t_{d_{2}}$ must be equal to $-(2 h(g-h-1)-1) /(2 g+1)$. On the other hand, we have

$$
\begin{aligned}
\phi_{g}\left(t_{d_{1}} t_{d_{2}}\right) & =\phi_{g}\left(\left(t_{c_{1}} \cdots t_{c_{2 h+1}}\right)^{2 h+2}\right) \\
& =(2 h+2) \phi_{g}\left(t_{c_{1}} \cdots t_{c_{2 h+1}}\right)-\sum_{i=1}^{2 h+1} \tau_{g}\left(\overline{c_{1} \cdots c_{2 h+1}}, \overline{\left(c_{1} \cdots c_{2 h+1}\right)^{i}}\right) \\
& =\frac{g+1}{2 g+1} \cdot(2 h+1)(2 h+2)-\sum_{i=1}^{2 h+1} \tau_{g}\left(\overline{c_{1} \cdots c_{2 h+1}}, \overline{\left(c_{1} \cdots c_{2 h+1}\right)^{i}}\right)
\end{aligned}
$$

from direct computations. Hence we conclude that

$$
\sum_{i=1}^{2 h+1} \tau_{g}\left(\overline{c_{1} \cdots c_{2 h+1}}, \overline{\left(c_{1} \cdots c_{2 h+1}\right)^{i}}\right)=2 h^{2}+4 h+1
$$

As a result, we obtain

$$
\begin{aligned}
c_{g}\left(C_{2 h+1, \mathrm{I}}\right)= & c_{g}\left(\left(c_{1} \cdots c_{2 h+1}\right)^{2 h+2} d_{1}^{-1} d_{2}^{-1}\right) \\
= & c_{g}\left(\left(c_{1} \cdots c_{2 h+1}\right)^{2 h+2}\right)-c_{g}\left(d_{1}\right)-c_{g}\left(d_{2}\right)-\tau_{g}\left(\overline{d_{1}}, \overline{d_{2}}\right) \\
= & (2 h+2) c_{g}\left(c_{1} \cdots c_{2 h+1}\right) \\
& +\sum_{i=1}^{2 h+1} \tau_{g}\left(\overline{c_{1} \cdots c_{2 h+1}}, \overline{\left(c_{1} \cdots c_{2 h+1}\right)^{i}}\right)-0-0-1 \\
= & (2 h+2) \cdot 0+\left(2 h^{2}+4 h+1\right)-1 \\
= & 2 h(h+2)
\end{aligned}
$$

and then

$$
I_{g}\left(C_{2 h+1, \mathrm{I}}\right)=-c_{g}\left(C_{2 h+1, \mathrm{I}}\right)-s\left(C_{2 h+1, \mathrm{I}}\right)=-2 h(h+2)-0=-2 h(h+2) .
$$

We have thus proved the proposition. 
Remark 3.11. T. Morifuji kindly told the authors that the value $\phi_{g}\left(t_{d_{1}} t_{d_{2}}\right)$ can directly be computed by using a method similar to one developed in his paper (see [30], Remark 2.5 and Example 2.6).

3.3. Lantern relation. Let $a$ and $b$ be simple closed curves on $\Sigma_{g}$ with $i(a, b)=2$ and algebraic intersection number 0 . We orient $a$ and $b$ locally on a neighborhood of each intersection point $p \in a \cap b$ such that the intersection number $(a \cdot b)_{p}$ at $p$ is +1 . Resolving all intersection points according to the local orientations, we obtain a new simple closed curve $c$. A regular neighborhood of $a \cup b$, which can be chosen to include $c$, is a genus- 0 subsurface $\Sigma$ of $\Sigma_{g}$ with 4 boundary components. We denote simple closed curves parallel to 4 boundary components of $\Sigma$ by $d_{1}, d_{2}, d_{3}$ and $d_{4}$. The relation

$$
t_{a} t_{b} t_{c}=t_{d_{1}} t_{d_{2}} t_{d_{3}} t_{d_{4}}
$$

is called the lantern relation, which was discovered by Dehn and rediscovered by Johnson [21]. We put

$$
L:=L(a, b)=a b c d_{1}^{-1} d_{2}^{-1} d_{3}^{-1} d_{4}^{-1} \in \mathcal{R} .
$$

The topological type of $L$ is not unique. We denote $L$ also by the following symbols according to the place where $L$ sits in $\Sigma_{g}$ :

$L_{\mathrm{I}}$ if all of $d_{1}, d_{2}, d_{3}$ and $d_{4}$ are non-separating and $\Sigma_{g}-\Sigma$ is connected;

$L_{\text {II }}$ if one of $d_{1}, d_{2}, d_{3}$ and $d_{4}$ is separating and the rest are non-separating;

$L_{\text {III }}$ if all of $d_{1}, d_{2}, d_{3}$ and $d_{4}$ are non-separating and $\Sigma_{g}-\Sigma$ is not connected;

$L_{\mathrm{IV}}$ if two of $d_{1}, d_{2}, d_{3}$ and $d_{4}$ are separating and the rest are non-separating;

$L_{\mathrm{V}}$ if all of $d_{1}, d_{2}, d_{3}$ and $d_{4}$ are separating.

Proposition 3.12. The signature $I_{g}(L)$ of a lantern relator $L$ is equal to +1 . In particular, it does not depend on the topological type of $L$.

Proof. From the definition of the map $c_{g}$, we have

$$
\begin{aligned}
c_{g}(L) & =\tau_{g}(\bar{a}, \bar{b})+\tau_{g}(\overline{a b}, \bar{c})+\tau_{g}\left(\overline{a b c}, \overline{d_{1}^{-1}}\right)+\tau_{g}\left(\overline{a b c d_{1}^{-1}}, \overline{d_{2}^{-1}}\right) \\
& +\tau_{g}\left(\overline{a b c d_{1}^{-1} d_{2}^{-1}}, \overline{d_{3}^{-1}}\right)+\tau_{g}\left(\overline{d_{4}}, \overline{d_{4}^{-1}}\right),
\end{aligned}
$$

where the last term $\tau_{g}\left(\overline{d_{4}}, \overline{d_{4}^{-1}}\right)$ is obviously equal to 0 . Carrying out explicit computation of $\tau_{g}$, we obtain the following table:

\begin{tabular}{|c||c|c|c|c|c|}
\hline type & $\tau_{g}(\bar{a}, \bar{b})$ & $\tau_{g}(\overline{a b}, \bar{c})$ & $\tau_{g}\left(\overline{a b c}, \overline{d_{1}^{-1}}\right)$ & $\tau_{g}\left(\overline{a b c d_{1}^{-1}}, \overline{d_{2}^{-1}}\right)$ & $\tau_{g}\left(\overline{\left.a b c d_{1}^{-1} d_{2}^{-1}, \overline{d_{3}^{-1}}\right)}\right.$ \\
\hline$L_{\mathrm{I}}$ & 0 & 0 & -1 & 0 & 0 \\
\hline$L_{\mathrm{II}}$ & 0 & +1 & -1 & 0 & 0 \\
\hline$L_{\mathrm{III}}$ & 0 & 0 & -1 & 0 & -1 \\
\hline$L_{\mathrm{IV}}$ & 0 & +1 & -1 & 0 & 0 \\
\hline$L_{\mathrm{V}}$ & 0 & 0 & 0 & 0 & 0 \\
\hline
\end{tabular}

Thus we have $c_{g}\left(L_{\mathrm{I}}\right)=-1, c_{g}\left(L_{\mathrm{III}}\right)=-2$ and $c_{g}\left(L_{\mathrm{II}}\right)=c_{g}\left(L_{\mathrm{IV}}\right)=c_{g}\left(L_{\mathrm{V}}\right)=0$. It is easy to see that $s\left(L_{\mathrm{I}}\right)=0, s\left(L_{\mathrm{III}}\right)=+1$ and $c_{g}\left(L_{\mathrm{II}}\right)=c_{g}\left(L_{\mathrm{IV}}\right)=c_{g}\left(L_{\mathrm{V}}\right)=-1$. Therefore we have $I_{g}(L)=+1$ in each case $L=L_{\mathrm{I}}, L_{\mathrm{II}}, L_{\mathrm{III}}, L_{\mathrm{IV}}, L_{\mathrm{V}}$. 
3.4. Star relation. Let $a_{1}, a_{2}, a_{3}$ and $b$ be simple closed curves on $\Sigma_{g}$ with $i\left(a_{i}, a_{j}\right)$ $=0(i \neq j)$ and $i\left(a_{i}, b\right)=1(i=1,2,3)$. A regular neighborhood of $a_{1} \cup a_{2} \cup a_{3} \cup b$ is a genus- 1 subsurface of $\Sigma_{g}$ with 3 boundary components. We denote simple closed curves parallel to 3 boundary components by $d_{1}, d_{2}$ and $d_{3}$. The relation

$$
t_{d_{1}} t_{d_{2}} t_{d_{3}}=\left(t_{a_{1}} t_{a_{2}} t_{a_{3}} t_{b}\right)^{3}
$$

is called the star relation, which was discovered by Gervais [16]. We put $E:=$ $\left(a_{1} a_{2} a_{3} b\right)^{3} d_{1}^{-1} d_{2}^{-1} d_{3}^{-1} \in \mathcal{R}$. The topological type of $E$ is not unique. We denote $E$ also by the following symbols according to the place where $E$ sits in $\Sigma_{g}$ :

$E_{\mathrm{I}}$ if all of $d_{1}, d_{2}$ and $d_{3}$ are non-separating;

$E_{\mathrm{II}}$ if one of $d_{1}, d_{2}$ and $d_{3}$ is separating and the rest are non-separating;

$E_{\mathrm{III}}$ if all of $d_{1}, d_{2}$ and $d_{3}$ are separating.

Proposition 3.13. The signature $I_{g}(E)$ of a star relator $E$ is equal to +5 . In particular, it does not depend on the topological type of $E$.

Proof. It follows from the proof of Theorem 1.6 of Gervais [16] that

$$
E_{\mathrm{I}} \equiv C_{3, \mathrm{I}}^{-1} L_{\mathrm{I}}^{-1}, E_{\mathrm{II}} \equiv C_{3, \mathrm{II}}^{-1} L_{\mathrm{III}}^{-1}, E_{\mathrm{III}} \equiv C_{3, \mathrm{II}}^{-1} L_{\mathrm{IV}}^{-1}\left(\bmod T_{0}, T_{1}, T_{2_{0}}\right) .
$$

We have values of $c_{g}$ on $E_{\mathrm{I}}, E_{\mathrm{II}}$ and $E_{\mathrm{III}}$.

$$
\begin{aligned}
c_{g}\left(E_{\mathrm{I}}\right) & =-c_{g}\left(C_{3, \mathrm{I}}\right)-c_{g}\left(L_{\mathrm{I}}\right)=-6-(-1)=-5, \\
c_{g}\left(E_{\mathrm{II}}\right) & =-c_{g}\left(C_{3, \mathrm{II}}\right)-c_{g}\left(L_{\mathrm{III}}\right)=-8-(-2)=-6, \\
c_{g}\left(E_{\mathrm{III}}\right) & =-c_{g}\left(C_{3, \mathrm{II}}\right)-c_{g}\left(L_{\mathrm{IV}}\right)=-8-0=-8 .
\end{aligned}
$$

It is easy to see that $s\left(E_{\mathrm{I}}\right)=0, s\left(E_{\mathrm{II}}\right)=+1$ and $s\left(E_{\mathrm{III}}\right)=+3$. Therefore we have $I_{g}(E)=+5$ in each case $E=E_{\mathrm{I}}, E_{\mathrm{II}}, E_{\mathrm{III}}$.

3.5. Hyperelliptic relation. The longest chain on $\Sigma_{g}$ is a chain $\left(c_{1}, \ldots, c_{2 g+1}\right)$ of length $2 g+1$. The relation

$$
\left(t_{c_{1}} \cdots t_{c_{2 g}} t_{c_{2 g+1}}^{2} t_{c_{2 g}} \cdots t_{c_{1}}\right)^{2}=1
$$

is called the hyperelliptic relation, which appeared in Birman-Hilden's paper [5]. We put $H_{g}=H\left(c_{1}, \ldots, c_{2 g+1}\right):=\left(c_{1} \cdots c_{2 g} c_{2 g+1}^{2} c_{2 g} \cdots c_{1}\right)^{2} \in \mathcal{R}$. The topological type of $H_{g}$ is unique.

Proposition 3.14. The signature $I_{g}\left(H_{g}\right)$ of a hyperelliptic relator $H_{g}$ is equal to $-4(g+1)$.

Proof. We have

$$
I_{g}\left(H_{g}\right)=-c_{g}\left(H_{g}\right)-s\left(H_{g}\right)=-4(g+1)-0=-4(g+1)
$$

from the formula (31) of Meyer [27].

The signature $I_{g}(\varrho)$ of a relator $\varrho \in \mathcal{R}$ is equal to that of another relator with the same topological type from Lemma 3.5. It seems to the authors that $I_{g}(\varrho)$ does not depend even on the topological type of $\varrho$ for arbitrary relator $\varrho \in \mathcal{R}$ because of Theorems 3.1 and 3.2 and Propositions 3.6, 3.9, 3.10, 3.12 and 3.13. 


\section{NON-HOLOMORPHIC LEFSCHETZ FIBRATIONS}

In this section we exhibit some known examples of Lefschetz fibrations and computations of their signatures using the function $I_{g}: \mathcal{R} \rightarrow \mathbb{Z}$. We also give some new examples of non-holomorphic Lefschetz fibrations over $S^{2}$.

We first review the definition and basic properties of Lefschetz fibrations. More details can be found in Matsumoto [29] and Gompf and Stipsicz [18.

Definition 4.1. Let $M$ and $B$ be compact oriented smooth manifolds of dimension 4 and 2, respectively. A smooth map $f: M \rightarrow B$ is called a Lefschetz fibration of genus $g$ if it satisfies the following conditions:

(i) $\partial M=f^{-1}(\partial B)$;

(ii) $f$ has finitely many critical values $b_{1}, \ldots, b_{n} \in \operatorname{int} B$ and $f$ is a smooth fiber bundle with fiber $\Sigma_{g}$ over $B-\left\{b_{1}, \ldots, b_{n}\right\}$;

(iii) for each $i(1 \leq i \leq n)$, there exists a unique critical point $p_{i}$ in the singular fiber $F_{i}:=f^{-1}\left(b_{i}\right)$ such that $f$ is locally written as $f\left(z_{1}, z_{2}\right)=z_{1}^{2}+z_{2}^{2}$ with respect to some local complex coordinates around $p_{i}$ and $b_{i}$ which are compatible with orientations of $M$ and $B$;

(iv) no fibers contain a $(-1)$-sphere.

We take a small disk $D_{i} \subset B$ centered at each $b_{i}$ and set $B_{0}:=B-\left(\operatorname{int} D_{1} \cup\right.$ $\left.\cdots \cup \operatorname{int} D_{n}\right)$ and $M_{0}:=f^{-1}\left(B_{0}\right)$. Since $f_{0}:=\left.f\right|_{B_{0}}: M_{0} \rightarrow B_{0}$ is a smooth fiber bundle with fiber $\Sigma_{g}$, we consider the homomorphism

$$
\chi: \pi_{1}\left(B_{0}\right) \rightarrow \pi_{1}\left(\mathrm{BDiff}_{+} \Sigma_{g}\right) \cong \pi_{0}\left(\operatorname{Diff}_{+} \Sigma_{g}\right)=\mathcal{M}_{g}
$$

induced by the classifying map $B_{0} \rightarrow$ BDiff $\Sigma_{g}$ of $f_{0} . \chi$ is called the holonomy homomorphism of $f_{0}$ (cf. Morita 31]). If $g \geq 2$, there exists a one-to-one correspondence between the isomorphism classes of the bundle $f_{0}$ and the conjugacy classes of the homomorphism $\chi$ by virtue of a result of Earle and Eells [11. Moreover, Matsumoto [29] proved that if $g \geq 2$, there exists a one-to-one correspondence between the isomorphism classes of the Lefschetz fibration $f: M \rightarrow B$ and the conjugacy classes of the homomorphism $\chi$ which sends each boundary curve to a right-handed Dehn twist along an essential simple closed curve on $\Sigma_{g}$.

Suppose that the base $B$ is the 2 -sphere $S^{2}$. Let $\gamma_{i}(i=1, \ldots, n)$ denote the loop consisting of $\partial D_{i}$ oriented clockwise and a path connecting a point on $\partial D_{i}$ to the base point $b_{0} \in \operatorname{int} B$. We choose these loops $\gamma_{1}, \ldots, \gamma_{n}$ so that the composition $\gamma_{1} \cdots \gamma_{n}$ is null-homotopic on $B_{0}$ and any two of them intersect only at $b_{0}$. Thus we obtain a presentation

$$
\pi_{1}\left(B_{0}\right)=\left\langle\gamma_{1}, \ldots, \gamma_{n} \mid \gamma_{1} \cdots \gamma_{n}=1\right\rangle
$$

of $\pi_{1}\left(B_{0}\right)$. For each $i, \chi\left(\gamma_{i}\right)$ is known to be a right-handed Dehn twist $t_{c_{i}}$ along some essential simple closed curve $c_{i}$ on $\Sigma_{g}$. Hence we have a positive relation

$$
t_{c_{1}} \cdots t_{c_{n}}=\chi\left(\gamma_{1} \cdots \gamma_{n}\right)=1 \in \mathcal{M}_{g}
$$

or a positive relator $c_{1} \cdots c_{n} \in \mathcal{R}$ associated to the Lefschetz fibration $f: M \rightarrow S^{2}$.

Theorem 4.2. Let $f: M \rightarrow S^{2}$ be a Lefschetz fibration of genus $g$ over $S^{2}$ and $c_{1} \cdots c_{n} \in \mathcal{R}$ a positive relator associated to $f$. Then the signature $\operatorname{Sign}(M)$ of the total space $M$ is equal to $I_{g}\left(c_{1} \cdots c_{n}\right)$ : namely

$$
\operatorname{Sign}(M)=I_{g}\left(c_{1} \cdots c_{n}\right) .
$$


Proof. It is an immediate consequence of Meyer's signature formula that $\operatorname{Sign}\left(M_{0}\right)$ $=-c_{g}\left(c_{1} \cdots c_{n}\right)$ because $f_{0}: M_{0} \rightarrow B_{0}$ is a fiber bundle over $B_{0}$ with fiber $\Sigma_{g}$ (see Meyer [27], Satz 1). The signature $\operatorname{Sign}\left(f^{-1}\left(D_{i}\right)\right)$ of a fibered neighborhood of the singular fiber $F_{i}$ is equal to 0 (resp. -1 ) if $c_{i}$ is non-separating (resp. separating). Hence we obtain

$$
\operatorname{Sign}(M)=-c_{g}\left(c_{1} \cdots c_{n}\right)-s\left(c_{1} \cdots c_{n}\right)=I_{g}\left(c_{1} \cdots c_{n}\right)
$$

from the Novikov additivity.

Although the positive relator $c_{1} \cdots c_{n} \in \mathcal{R}$ actually depends on a choice of a loop system $\left(\gamma_{1}, \ldots, \gamma_{n}\right)$ on $B_{0}$, its equivalence class modulo conjugations of all elements $c_{1}, \ldots, c_{n}$ by a fixed element of $\mathcal{F}$ and elementary transformations

$$
\begin{gathered}
c_{1} \cdots c_{i} c_{i+1} \cdots c_{n} \sim c_{1} \cdots c_{i+1}\left(c_{i}\right)_{c_{i+1}^{-1}} \cdots c_{n}, \\
c_{1} \cdots c_{i} c_{i+1} \cdots c_{n} \sim c_{1} \cdots\left(c_{i+1}\right)_{c_{i}} c_{i} \cdots c_{n},
\end{gathered}
$$

where $(a)_{b}=b a b^{-1}$ and $i=1, \ldots, n-1$, is uniquely determined by the isomorphism class of the Lefschetz fibration $f: M \rightarrow S^{2}$. Conversely, any positive relator $\varrho \in \mathcal{R}$ can be realized as a relator associated to some Lefschetz fibration over $S^{2}$. It is worth noting that $(a)_{b}=b a b^{-1}$ is not in $\mathcal{S}$ but in $\mathcal{F}$ for $a, b \in \mathcal{S}$. We think of $(a)_{b}$ as the element $t_{b}(a)$ of $\mathcal{S}$ because of the braid relation $t_{t_{b}(a)}=t_{b} t_{a} t_{b}^{-1}$.

Precisely speaking, the elementary transformation

$$
c_{1} \cdots c_{i} c_{i+1} \cdots c_{n} \sim c_{1} \cdots\left(c_{i+1}\right)_{c_{i}} c_{i} \cdots c_{n}=c_{1} \cdots t_{c_{i}}\left(c_{i+1}\right) c_{i} \cdots c_{n}
$$

is the composition of inserting a trivial relator $c_{i}^{-1} c_{i} \in \mathcal{R}$ and applying $T\left(c_{i+1}, c_{i}\right)^{-1}$ substitution.

Theorem 4.3. Let $f: M \rightarrow S^{2}, f^{\prime}: M^{\prime} \rightarrow S^{2}$ be Lefschetz fibrations of genus $g$ over $S^{2}$ and $\varsigma, \varsigma^{\prime} \in \mathcal{R}$ positive relators associated to $f, f^{\prime}$, respectively. Suppose that a $\varrho$-substitution to $\varsigma$ yields $\varsigma^{\prime}$ for some relator $\varrho \in \mathcal{R}$. Then we have

$$
\operatorname{Sign}\left(M^{\prime}\right)=\operatorname{Sign}(M)+I_{g}(\varrho) .
$$

Proof. Straightforward from Theorem 4.2 and Lemma 3.5.

The two theorems above are generalized to those for Lefschetz fibrations over closed surfaces of arbitrary genus. As a consequence, we conclude that signatures of Lefschetz fibrations corresponding to relators obtained in $\S 3$ of [13] do not depend on topological types of relators.

Notation 4.4. We denote the signature and the Euler characteristic of a compact oriented smooth 4-manifold $M$ by $\sigma=\operatorname{Sign}(M)$ and $e$, respectively. For an almost complex closed 4-manifold $M$, we set $\chi_{h}:=(\sigma+e) / 4$ (the holomorphic Euler characteristic) and $K^{2}:=3 \sigma+2 e$. We define relative numerical invariants $\chi_{f}:=$ $\chi_{h}+g-1, K_{f}^{2}:=K^{2}+8(g-1)$ and the slope $\lambda_{f}:=K_{f}^{2} / \chi_{f}$ for a Lefschetz fibration $f: M \rightarrow B$ of genus $g$ over a closed surface $B$. It is easily seen that $e=-4(g-1)+n$ for a Lefschetz fibration $f: M \rightarrow S^{2}$ of genus $g$ with $n$ singular fibers.

4.1. Three hyperelliptic Lefschetz fibrations. We shall begin signature computations with the following well-known Lefschetz fibrations.

Let $\left(c_{1}, \ldots, c_{2 g+1}\right)$ be a chain of length $2 g+1$ on $\Sigma_{g}$. Since a hyperelliptic relator $H_{g}=\left(c_{1} \cdots c_{2 g} c_{2 g+1}^{2} c_{2 g} \cdots c_{1}\right)^{2}$ of genus $g$ is a positive relator, we obtain 
a Lefschetz fibration $M_{1} \rightarrow S^{2}$ of genus $g$ corresponding to $H_{g}$. A chain relator $C_{2 g+1}=\left(c_{1} \cdots c_{2 g+1}\right)^{2 g+2} d_{1}^{-1} d_{2}^{-1}$ of length $2 g+1$ combined with two identity relators $A\left(d_{1}\right)=d_{1}, A\left(d_{2}\right)=d_{2}$ is a positive relator $C_{2 g+1} A\left(d_{2}\right) A\left(d_{1}\right)=$ $\left(c_{1} \cdots c_{2 g+1}\right)^{2 g+2}$. A chain relator $C_{2 g}=\left(c_{1} \cdots c_{2 g}\right)^{4 g+2} d^{-1}$ of length $2 g$ combined with an identity relator $A(d)=d$ is a positive relator $C_{2 g} A(d)=\left(c_{1} \cdots c_{2 g}\right)^{4 g+2}$. We have Lefschetz fibrations $M_{2} \rightarrow S^{2}$ and $M_{3} \rightarrow S^{2}$ corresponding to $C_{2 g+1} A\left(d_{2}\right) A\left(d_{1}\right)$ and $C_{2 g} A(d)$, respectively. By virtue of Theorem 4.2 and 4.3 , signatures of $M_{1}, M_{2}$ and $M_{3}$ are equal to $I_{g}\left(H_{g}\right), I_{g}\left(C_{2 g+1}\right)+2 I_{g}(A)$ and $I_{g}\left(C_{2 g}\right)+I_{g}(A)$, respectively. Invariants of $M_{1}, M_{2}$ and $M_{3}$ are calculated as in the following table:

\begin{tabular}{|c||c|c|c|c|}
\hline 4-manifold & $\sigma$ & $e$ & $\chi_{h}$ & $K^{2}$ \\
\hline$M_{1}$ & $-4(g+1)$ & $4(g+2)$ & 1 & $-4(g-1)$ \\
\hline$M_{2}$ & $-2(g+1)^{2}$ & $2\left(2 g^{2}+g+3\right)$ & $g(g-1) / 2+1$ & $2(g-1)(g-3)$ \\
\hline$M_{3}$ & $-4 g(g+1)$ & $4\left(2 g^{2}+1\right)$ & $g^{2}-g+1$ & $4(g-1)(g-2)$ \\
\hline
\end{tabular}

Each of $M_{1}, M_{2}$ and $M_{3}$ admits a (-1)-section, then cannot be decomposed to any non-trivial fiber sum and is a simply connected non-spin 4-manifold (cf. Stipsicz 39], Theorem 1.3, Siebert and Tian [35], Proposition 3.2, and Stipsicz [40], Theorem 1.3). If $g=1$, all of them are the 4-manifold $E(1)$, which is $\mathbb{C} P^{2} \sharp 9 \overline{\mathbb{C} P}^{2}$ equipped with an elliptic fibration. If $g=2, M_{1}, M_{2}$ and $M_{3}$ are $\mathbb{C} P^{2} \sharp 13 \overline{\mathbb{C P}}^{2}, E(2) \sharp 2 \overline{\mathbb{C P}}^{2}$ and $H^{\prime}(1)$, respectively (see Gompf and Stipsicz [18 for notation) and are studied minutely by Matsumoto [29] as Examples A, C and D. According to Ito [20], $M_{1}$ can be obtained by splitting singular fibers of a certain holomorphic fibration $f_{g}$ : $M_{g} \rightarrow S^{2}$ of genus $g$ and is diffeomorphic to $\mathbb{C} P^{2} \sharp(4 g+5) \overline{\mathbb{C P}}^{2}$ for arbitrary genus $g$.

A fiber sum of copies of $M_{1}, M_{2}$ and $M_{3}$ are isomorphic to one of $\sharp_{F} n M_{1}$, $M_{2} \sharp_{F}(n-1) M_{1}$ and $\sharp_{F} n M_{3}$, where $\sharp_{F}$ stands for the fiber sum. $\sharp_{F} n M_{1}$ and $M_{2} \sharp_{F}(n-1) M_{1}$ are spin if and only if $g$ is odd and $n$ is even. $\sharp_{F} n M_{3}$ is spin if and only if $n$ is even (see Nagami [32] and Stipsicz [40]).

The slope $\lambda_{f}$ of these three fibrations and their fiber sums are equal to $4-4 / g$, which is known as the lower bound of the slope of relatively minimal holomorphic fibrations of curves of genus $g$ on non-singular algebraic surfaces (see Xiao [45]).

Remark 4.5. The three Lefschetz fibrations above are all hyperelliptic in the sense of [12. Their signature can also be computed by using the local signature for hyperelliptic fibrations (see [2], [3], 12], 35] and [36]).

4.2. Cadavid-Korkmaz's examples. Matsumoto's genus-2 Lefschetz fibration (Example B of [29]) were generalized to that of arbitrary genus $g$ independently by Cadavid [7] and Korkmaz [25].

We have constructed a positive relator $C_{2 g+1} A\left(d_{2}\right) A\left(d_{1}\right)=\left(c_{1} \cdots c_{2 g+1}\right)^{2 g+2}$ in $\S 4.1$. If we put

$$
\Gamma:=\left(c_{1} c_{2} \cdots c_{g}\right) \cdots\left(c_{1} c_{2} c_{3}\right)\left(c_{1} c_{2}\right) c_{1}, \quad \Delta:=\left(c_{1} c_{2} \cdots c_{2 g+1}\right) \Gamma,
$$

then we have $\Gamma^{-1} C_{2 g+1} A\left(d_{2}\right) A\left(d_{1}\right) \Gamma \equiv \Delta^{2}\left(\bmod T_{0}, T_{1}\right)$. We can take certain simple closed curves $B_{0}, B_{1}, \ldots, B_{g}$ to verify

$$
\Delta \equiv B_{0} B_{1} \cdots B_{g}\left(c_{g} \cdots c_{2} c_{1}\right)^{2 g+2} \quad\left(\bmod T_{0}, T_{1}\right)
$$

(see Korkmaz [25], Figure 2 and Theorem 2.4). Consider the chain relator $C_{g}=$ $C\left(c_{1}, \ldots, c_{g}\right)$ of length $g$ and let $c$ (resp. $\left.a, b\right)$ be simple closed curve(s) parallel to 
the boundary of a regular neighborhood of $C_{g}$ in $\Sigma_{g}$ when $g$ is even (resp. odd). Applying $C_{g}^{-1}$-substitutions to $B_{0} B_{1} \cdots B_{g}\left(c_{g} \cdots c_{2} c_{1}\right)^{2 g+2}$, we obtain

$$
\begin{aligned}
\Delta C_{g}^{-1} & \equiv B_{0} B_{1} \cdots B_{g} c\left(\bmod T_{0}, T_{1}\right) \quad(g: \text { even }), \\
\Delta C_{g}^{-2} & \equiv B_{0} B_{1} \cdots B_{g} a^{2} b^{2}\left(\bmod T_{0}, T_{1}\right) \quad(g: \text { odd }) .
\end{aligned}
$$

Therefore we have a positive relator

$$
C K_{g}:=\left\{\begin{array}{ll}
\left(B_{0} B_{1} \cdots B_{g} c\right)^{2} & (g: \text { even }) \\
\left(B_{0} B_{1} \cdots B_{g} a^{2} b^{2}\right)^{2} & (g: \text { odd })
\end{array} \in \mathcal{R}\right.
$$

and the corresponding Lefschetz fibration $M^{C K} \rightarrow S^{2}$ of genus $g$.

The signature of $M^{C K}$ is easily computed from signatures of chain relators by virtue of Theorem 4.2 and 4.3 :

$$
\begin{aligned}
\operatorname{Sign}\left(M^{C K}\right) & = \begin{cases}I_{g}\left(C_{2 g+1}\right)+2 I_{g}(A)-2 I_{g}\left(C_{g}\right) & (g: \text { even }), \\
I_{g}\left(C_{2 g+1}\right)+2 I_{g}(A)-4 I_{g}\left(C_{g}\right) & (g: \text { odd }),\end{cases} \\
& = \begin{cases}-4 & (g: \text { even }), \\
-8 & (g: \text { odd }) .\end{cases}
\end{aligned}
$$

Invariants of $M^{C K}$ are calculated as in the following table:

\begin{tabular}{|c||c|c|c|c|c|}
\hline 4-manifold & $\sigma$ & $e$ & $\chi_{h}$ & $K^{2}$ & $\lambda_{f}$ \\
\hline$M^{C K}(g:$ even $)$ & -4 & $8-2 g$ & $1-g / 2$ & $4-4 g$ & $8(g-1) / g$ \\
\hline$M^{C K}(g$ : odd $)$ & -8 & $14-2 g$ & $(3-g) / 2$ & $4-4 g$ & $8(g-1) /(g+1)$ \\
\hline
\end{tabular}

The Lefschetz fibration $M^{C K}$ admits a (-1)-section and cannot be decomposed to a non-trivial fiber sum. $M^{C K}$ is known to be diffeomorphic to $\Sigma_{g / 2} \times S^{2} \sharp 4 \overline{\mathbf{C P}}^{2}$ (resp. $\Sigma_{(g-1) / 2} \times S^{2} \sharp 8 \overline{\mathbf{C P}}^{2}$ ) if $g$ is even (resp. odd) (see Matsumoto [29], Stipsicz [40] and Korkmaz [25]). $M^{C K} \rightarrow S^{2}$ is hyperelliptic if $g$ is even while it neither is it if $g$ is odd.

4.3. A generalization of Fuller's construction. Fuller gave an example of nonhyperelliptic Lefschetz fibration of genus 3 (see Ozbagci [33] and Smith [37]). We generalize his example to that of arbitrary genus $g$ and show that some of them are not isomorphic to any holomorphic Lefschetz fibration.

We construct an "odd subchain part" in a longer chain relator using braid relations and apply $C$-substitutions to it in order to destroy hyperellipticity.

We first need to show the following lemma.

Lemma 4.6. Let $\left(c_{1}, \ldots, c_{n}\right)$ be a chain of length $n$ on $\Sigma_{g}$. Then we have the following equivalence:

$$
\begin{aligned}
\left(c_{1} c_{2} \cdots c_{n}\right)^{k+1} \equiv & \left(c_{1} c_{2} \cdots c_{k}\right)^{k+1}\left(c_{k+1} c_{k} \cdots c_{2} c_{1}\right)\left(c_{k+2} c_{k+1} \cdots c_{3} c_{2}\right) \\
& \cdots\left(c_{n} c_{n-1} \cdots c_{n-k+1} c_{n-k}\right) \quad\left(\bmod T_{0}, T_{1}\right)
\end{aligned}
$$

for $k=1, \ldots, n-1$.

Proof. Fix an integer $k$ such that $1 \leq k \leq n-1$. We prove the next equivalence by induction on $i$,

$$
\begin{aligned}
\left(c_{1} c_{2} \cdots c_{n}\right)^{k+1} \equiv & \left(c_{1} c_{2} \cdots c_{k}\right)^{i}\left(c_{k+1} c_{k} \cdots c_{k-i+2}\right)\left(c_{k+2} c_{k+1} \cdots c_{k-i+3}\right) \\
& \cdots\left(c_{n} c_{n-1} \cdots c_{n-i+1}\right)\left(c_{1} c_{2} \cdots c_{n}\right)^{k-i+1} \quad\left(\bmod T_{0}, T_{1}\right)
\end{aligned}
$$


for $i=1, \ldots, k+1$. It is trivial for $i=1$. Suppose that it is verified for $i$. We use

$(*) \quad \equiv c_{j+k-n} \cdot\left(c_{k+1} c_{k} \cdots c_{k-i+1}\right)\left(c_{k+2} c_{k+1} \cdots c_{k-i+2}\right) \cdots\left(c_{n} c_{n-1} \cdots c_{n-i}\right)$

$\left(\bmod T_{0}, T_{1}\right)$

for $j=n-i+1, \cdots, n$ (see Korkmaz [25], Lemma 2.1) to show

$$
\begin{aligned}
& \left(c_{k+1} c_{k} \cdots c_{k-i+2}\right)\left(c_{k+2} c_{k+1} \cdots c_{k-i+3}\right) \cdots\left(c_{n} c_{n-1} \cdots c_{n-i+1}\right) c_{1} c_{2} \cdots c_{n} \\
& \equiv c_{1} c_{2} \cdots c_{k-i}\left(c_{k+1} c_{k} \cdots c_{k-i+2}\right)\left(c_{k+2} c_{k+1} \cdots c_{k-i+3}\right) \cdots\left(c_{n} c_{n-1} \cdots c_{n-i+1}\right) \\
& \cdot c_{k-i+1} c_{k-i+2} \cdots c_{n} \\
& \equiv c_{1} c_{2} \cdots c_{k-i}\left(c_{k+1} c_{k} \cdots c_{k-i+1}\right)\left(c_{k+2} c_{k+1} \cdots c_{k-i+2}\right) \cdots\left(c_{n} c_{n-1} \cdots c_{n-i}\right) \\
& \text { - } c_{n-i+1} c_{n-i+2} \cdots c_{n} \\
& \underset{(*)}{\equiv} c_{1} c_{2} \cdots c_{k-i+1}\left(c_{k+1} c_{k} \cdots c_{k-i+1}\right)\left(c_{k+2} c_{k+1} \cdots c_{k-i+2}\right) \cdots\left(c_{n} c_{n-1} \cdots c_{n-i}\right) \\
& \cdot c_{n-i+2} c_{n-i+3} \cdots c_{n} \\
& \equiv \cdots \\
& (*) \\
& \underset{(*)}{\equiv} c_{1} c_{2} \cdots c_{k}\left(c_{k+1} c_{k} \cdots c_{k-i+1}\right)\left(c_{k+2} c_{k+1} \cdots c_{k-i+2}\right) \cdots\left(c_{n} c_{n-1} \cdots c_{n-i}\right)
\end{aligned}
$$

$\left(\bmod T_{0}, T_{1}\right)$.

Then we obtain

$$
\begin{aligned}
\left(c_{1} c_{2} \cdots c_{n}\right)^{k+1} \equiv & \left(c_{1} c_{2} \cdots c_{k}\right)^{i+1}\left(c_{k+1} c_{k} \cdots c_{k-i+1}\right)\left(c_{k+2} c_{k+1} \cdots c_{k-i+2}\right) \\
& \cdots\left(c_{n} c_{n-1} \cdots c_{n-i}\right)\left(c_{1} c_{2} \cdots c_{n}\right)^{k-i}\left(\bmod T_{0}, T_{1}\right) .
\end{aligned}
$$

Thus we have proven the given equivalence.

We have obtained positive relators $C_{2 g+1} A\left(d_{2}\right) A\left(d_{1}\right)=\left(c_{1} \cdots c_{2 g+1}\right)^{2 g+2}$ and $C_{2 g} A(d)=\left(c_{1} \cdots c_{2 g}\right)^{4 g+2}$ in $\S 4.1$.

For $h=0, \ldots, g-1$, we set $n=2 g+1,2 g, k=2 h+1$ in Lemma 4.6. Then we have

$$
\begin{aligned}
C_{2 g+1} A\left(d_{2}\right) A\left(d_{1}\right) \equiv & \left(c_{1} c_{2} \cdots c_{2 h+1}\right)^{2 h+2}\left(c_{2 h+2} c_{2 h+1} \cdots c_{2} c_{1}\right)\left(c_{2 h+3} c_{2 h+2} \cdots c_{3} c_{2}\right) \\
& \cdots\left(c_{2 g+1} c_{2 g} \cdots c_{2 g-2 h+1} c_{2 g-2 h}\right)\left(c_{1} c_{2} \cdots c_{2 g+1}\right)^{2 g-2 h} \\
C_{2 g} A(d) \equiv & \left(c_{1} c_{2} \cdots c_{2 h+1}\right)^{2 h+2}\left(c_{2 h+2} c_{2 h+1} \cdots c_{2} c_{1}\right)\left(c_{2 h+3} c_{2 h+2} \cdots c_{3} c_{2}\right) \\
& \cdots\left(c_{2 g} c_{2 g-1} \cdots c_{2 g-2 h} c_{2 g-2 h-1}\right)\left(c_{1} c_{2} \cdots c_{2 g}\right)^{4 g-2 h} \\
& \left(\bmod T_{0}, T_{1}\right) .
\end{aligned}
$$

Applying $C_{2 h+1}^{-1}$-substitutions to words in right-hand sides, we get positive relators

$$
\begin{aligned}
F_{h}^{\text {odd }}:= & d_{h, 1} d_{h, 2}\left(c_{2 h+2} c_{2 h+1} \cdots c_{2} c_{1}\right)\left(c_{2 h+3} c_{2 h+2} \cdots c_{3} c_{2}\right) \\
& \cdots\left(c_{2 g+1} c_{2 g} \cdots c_{2 g-2 h+1} c_{2 g-2 h}\right)\left(c_{1} c_{2} \cdots c_{2 g+1}\right)^{2 g-2 h}, \\
F_{h}^{\text {even }}:= & d_{h, 1} d_{h, 2}\left(c_{2 h+2} c_{2 h+1} \cdots c_{2} c_{1}\right)\left(c_{2 h+3} c_{2 h+2} \cdots c_{3} c_{2}\right) \\
& \cdots\left(c_{2 g} c_{2 g-1} \cdots c_{2 g-2 h} c_{2 g-2 h-1}\right)\left(c_{1} c_{2} \cdots c_{2 g}\right)^{4 g-2 h}
\end{aligned}
$$

where $d_{h, 1}$ and $d_{h, 2}$ are simple closed curves parallel to the boundary of a regular neighborhood of the chain $\left(c_{1}, \ldots, c_{2 h+1}\right)$. 
Let $M_{h}^{\text {odd }}$ and $M_{h}^{\text {even }}$ be Lefschetz fibrations of genus $g$ over $S^{2}$ corresponding to positive relators $F_{h}^{\text {odd }}$ and $F_{h}^{\text {even }}$, respectively. Signatures of $M_{h}^{\text {odd }}$ and $M_{h}^{\text {even }}$ are easily computed from signatures of chain relators by virtue of Theorem 4.2 and 4.3:

$$
\begin{aligned}
\operatorname{Sign}\left(M_{h}^{\text {odd }}\right) & =I_{g}\left(C_{2 g+1}\right)+2 I_{g}(A)-I_{g}\left(C_{2 h+1}\right) \\
& =-2(g+1)^{2}+2 h(h+2) \\
\operatorname{Sign}\left(M_{h}^{\text {even }}\right) & =I_{g}\left(C_{2 g}\right)+I_{g}(A)-I_{g}\left(C_{2 h+1}\right) \\
& =-4 g(g+1)+2 h(h+2)
\end{aligned}
$$

$M_{h}^{\text {odd }}$ and $M_{h}^{\text {even }}$ are simply connected non-spin 4-manifolds. Other invariants of them are calculated as in the following table:

\begin{tabular}{|c||c|c|}
\hline 4-manifold & $e$ & $\lambda_{f}$ \\
\hline$M_{h}^{\text {odd }}$ & $2\left(2 g^{2}+g-2 h^{2}-3 h+3\right)$ & $4\left(g^{2}-h^{2}-1\right) /(g(g+1)-h(h+1))$ \\
\hline$M_{h}^{\text {even }}$ & $2\left(4 g^{2}-2 h^{2}-3 h+2\right)$ & $4\left(2 g^{2}-2 g-h^{2}\right) /\left(2 g^{2}-h(h+1)\right)$ \\
\hline
\end{tabular}

Proposition 4.7. Lefschetz fibrations $M_{1}^{\text {odd }}$ and $M_{1}^{\text {even }}$ of genus 3 , Lefschetz fibrations $M_{1}^{\text {odd }}, M_{2}^{\text {odd }}, M_{1}^{\text {even }}$ and $M_{2}^{\text {even }}$ of genus 4 and Lefschetz fibrations $M_{1}^{\text {odd }}$, $M_{2}^{\text {odd }}, M_{3}^{\text {odd }}, M_{1}^{\text {even }}, M_{2}^{\text {even }}$ and $M_{3}^{\text {even }}$ of genus 5 are not isotopic to any holomorphic Lefschetz fibration over $\mathbb{C} P^{1}$.

Proof. Lefschetz fibrations exhibited above are not hyperelliptic in the sense of [12] because they should have fractional signature from the local signature formula if they were hyperelliptic. If a non-hyperelliptic Lefschetz fibration is isotopic to a holomorphic fibration, the slope $\lambda_{f}$ must be greater than or equal to 3 (resp. 24/7,40/11) when $g=3$ (resp. $g=4,5$ ) by virtue of theorems of Konno [23], 24] and Chen [8]. On the other hand, the slope $\lambda_{f}$ is equal to $14 / 5$ and $11 / 4$ for $M_{1}^{\text {odd }}$ and $M_{1}^{\text {even }}$ of genus 3 , to $28 / 9,22 / 7,46 / 15$ and $40 / 13$ for $M_{1}^{\text {odd }}, M_{2}^{\text {odd }}, M_{1}^{\text {even }}$ and $M_{2}^{\text {even }}$ of genus 4 and $23 / 7,10 / 3,10 / 3,13 / 4,36 / 11$ and $62 / 19$ for $M_{1}^{\text {odd }}, M_{2}^{\text {odd }}, M_{3}^{\text {odd }}, M_{1}^{\text {even }}, M_{2}^{\text {even }}$ and $M_{3}^{\text {even }}$ of genus 5 , respectively. This completes the proof.

For every $g \geq 3$ and $h=0, \ldots, g-1, M_{h}^{\text {odd }}$ and $M_{h}^{\text {even }}$ satisfies inequalities $4-4 / g \leq \lambda_{f} \leq 4$. The equality $\lambda_{f}=4-4 / g$ holds if and only if $h=g-1$, which is the only case that $M_{h}^{\text {odd }}$ and $M_{h}^{\text {even }}$ are hyperelliptic (see Xiao [45] and Konno [24]).

Remark 4.8. The Lefschetz fibration $M_{1}^{\text {even }} \rightarrow S^{2}$ of genus 3 is nothing but Fuller's original example. Smith [37] deduced an inequality equivalent to $\lambda_{f} \geq 3$ from study of divisors in moduli space and proved non-holomorphicity for this fibration.

4.4. Lefschetz fibrations coming from lantern relations. The lantern relation is essentially non-hyperelliptic. We introduce new type examples of nonhyperelliptic Lefschetz fibrations coming from lantern relations.

We first construct an example peculiar to genus 3. The idea of this example is to construct a "lantern part" in a chain of length 7 . We recall the positive relator $C_{7} A\left(d_{2}\right) A\left(d_{1}\right)=\left(c_{1} \cdots c_{7}\right)^{8}$ on the genus-3 surface $\Sigma_{3}$ obtained in $\S 4.1$. 
Lemma 4.9. For a chain $\left(c_{1}, \ldots, c_{7}\right)$ of length 7 on $\Sigma_{3}$, we have the following equivalence:

$$
\begin{aligned}
& \left(c_{1} c_{2} c_{3} c_{4} c_{5} c_{6} c_{7}\right)^{4} \\
\equiv & c_{4} \cdot c_{1} c_{2} c_{3} c_{4} c_{5} \cdot c_{1} c_{2} c_{3} c_{4} c_{5} c_{6} \cdot c_{1} c_{3} c_{5} c_{7} \cdot c_{2} c_{3} c_{4} c_{5} c_{6} c_{7} \cdot c_{3} c_{4} c_{5} c_{6} c_{7} \cdot c_{4} \\
& \quad\left(\bmod T_{0}, T_{1}\right) .
\end{aligned}
$$

Proof. We apply $T_{0^{-}}$and $T_{1}$-substitutions to the left-hand side repeatedly:

$$
\begin{aligned}
& \left(c_{1} c_{2} c_{3} c_{4} c_{5} c_{6} c_{7}\right)^{4} \\
= & c_{1} c_{2} c_{3} c_{4} c_{5} c_{6} c_{7} \cdot c_{1} c_{2} c_{3} c_{4} c_{5} c_{6} c_{7} \cdot c_{1} c_{2} c_{3} c_{4} c_{5} c_{6} c_{7} \cdot c_{1} c_{2} c_{3} c_{4} c_{5} c_{6} c_{7} \\
\equiv & c_{1} c_{2} c_{3} c_{4} c_{5} c_{6} \cdot c_{1} c_{2} c_{3} c_{4} c_{5} \cdot c_{7} c_{6} c_{7} \cdot c_{1} c_{2} c_{1} \cdot c_{3} c_{4} c_{5} c_{6} c_{7} \cdot c_{2} c_{3} c_{4} c_{5} c_{6} c_{7} \\
\equiv & c_{1} c_{2} c_{3} c_{4} c_{5} \cdot c_{1} c_{2} c_{3} c_{4} \cdot c_{6} c_{5} c_{6} \cdot c_{7} c_{6} \cdot c_{2} c_{1} \cdot c_{2} c_{3} c_{2} \cdot c_{4} c_{5} c_{6} c_{7} \cdot c_{3} c_{4} c_{5} c_{6} c_{7} \\
\equiv & c_{1} c_{2} c_{3} c_{4} c_{5} \cdot c_{1} \cdot c_{2} c_{3} c_{2} \cdot c_{4} c_{5} c_{6} \cdot c_{5} c_{7} \cdot c_{1} c_{3} \cdot c_{2} c_{3} c_{4} \cdot c_{6} c_{5} c_{6} \cdot c_{7} \cdot c_{3} c_{4} c_{5} c_{6} c_{7} \\
\equiv & c_{1} c_{2} \cdot c_{4} c_{3} c_{4} \cdot c_{5} \cdot c_{1} c_{2} c_{3} c_{4} c_{5} c_{6} \cdot c_{1} c_{3} c_{5} c_{7} \cdot c_{2} c_{3} c_{4} c_{5} c_{6} c_{7} \cdot c_{3} \cdot c_{5} c_{4} c_{5} \cdot c_{6} c_{7} \\
\equiv & c_{4} \cdot c_{1} c_{2} c_{3} c_{4} c_{5} \cdot c_{1} c_{2} c_{3} c_{4} c_{5} c_{6} \cdot c_{1} c_{3} c_{5} c_{7} \cdot c_{2} c_{3} c_{4} c_{5} c_{6} c_{7} \cdot c_{3} c_{4} c_{5} c_{6} c_{7} \cdot c_{4} \\
& \quad\left(\bmod T_{0}, T_{1}\right) .
\end{aligned}
$$

Thus the lemma has been proven.

The complement of an open regular neighborhood of the curves $c_{1}, c_{3}, c_{5}, c_{7}$ is homeomorphic to two copies of $\Sigma_{0,4}$. Take one of two components and draw simple closed curves $a, b$ and $c$ on it as in $\S 3.2$ so that $d_{1}, d_{2}, d_{3}$ and $d_{4}$ coincide with $c_{1}, c_{3}, c_{5}$ and $c_{7}$. Put $L:=a b c c_{1}^{-1} c_{3}^{-1} c_{5}^{-1} c_{7}^{-1} \in \mathcal{R}$. Applying $L$-substitution to the right-hand side of the equivalence in Lemma 4.9, we obtain a positive relator

$$
U:=c_{4} \cdot c_{1} \cdots c_{5} \cdot c_{1} \cdots c_{6} \cdot a b c \cdot c_{2} \cdots c_{7} \cdot c_{3} \cdots c_{7} \cdot c_{4}\left(c_{1} \cdots c_{7}\right)^{4}
$$

from the positive relator $C_{7} A\left(d_{2}\right) A\left(d_{1}\right)=\left(c_{1} \cdots c_{7}\right)^{8}$ and the corresponding Lefschetz fibration $M^{U} \rightarrow S^{2}$ of genus 3 .

We next treat both chain and lantern relations to construct another example. The idea of this construction is to connect two chains using a lantern as a connector.

Lemma 4.10. Let $\left(c_{1}, \ldots, c_{n}\right)$ be a chain of length $n$ on $\Sigma_{g}$. Then we have the following equivalence:

$$
\begin{aligned}
\left(c_{1} c_{2} \cdots c_{n}\right)^{k+1} \equiv & \left(c_{k+1} c_{k} \cdots c_{1}\right)\left(c_{k+2} c_{k+1} \cdots c_{2}\right) \cdots\left(c_{n} c_{n-1} \cdots c_{n-k}\right) \\
& \cdot\left(c_{n-k+1} c_{n-k+2} \cdots c_{n}\right)^{k+1}\left(\bmod T_{0}, T_{1}\right)
\end{aligned}
$$

for $k=0, \ldots, n-1$.

Proof. Similar to the proof of Lemma 4.6.

Let $\left(c_{1}, \ldots, c_{2 g+1}\right)$ be a chain of length $2 g+1$ on $\Sigma_{g}$ and $C_{2 g} A:=\left(c_{1} c_{2} \cdots c_{2 g}\right)^{4 g+2}$ and $\bar{C}_{2 g} A:=\left(c_{2} c_{3} \cdots c_{2 g+1}\right)^{4 g+2}$ positive relators defined as in $\S 4.1$. For $h=$ $2, \ldots, g-2$, we have constructed the positive relator

$$
\begin{aligned}
F_{h-1}^{\text {even }}:= & d_{h-1,1} d_{h-1,2}\left(c_{2 h} c_{2 h-1} \cdots c_{2} c_{1}\right)\left(c_{2 h+1} c_{2 h} \cdots c_{3} c_{2}\right) \\
& \cdots\left(c_{2 g} c_{2 g-1} \cdots c_{2 g-2 h+2} c_{2 g-2 h+1}\right)\left(c_{1} c_{2} \cdots c_{2 g}\right)^{4 g-2 h+2}
\end{aligned}
$$


from $C_{2 g} A$ using $C_{2 h-1}^{-1}$-substitution in $\S 4.3$. We apply Lemma 4.10 to $\bar{C}_{2 g} A$ and obtain the following equivalence:

$$
\begin{aligned}
\bar{C}_{2 g} A \equiv & \left(c_{2} c_{3} \cdots c_{2 g+1}\right)^{2 g+2 h+2}\left(c_{2 g-2 h+1} \cdots c_{3} c_{2}\right)\left(c_{2 g-2 h+2} \cdots c_{4} c_{3}\right) \\
& \cdots\left(c_{2 g+1} \cdots c_{2 h+3} c_{2 h+2}\right)\left(c_{2 h+3} c_{2 h+4} \cdots c_{2 g+1}\right)^{2 g-2 h}
\end{aligned}
$$

$\left(\bmod T_{0}, T_{1}\right)$.

Let $d_{h, 1}, d_{h, 2}$ be simple closed curves parallel to the boundary of a regular neighborhood of the chain $\left(c_{2 h+3} c_{2 h+4} \cdots c_{2 g+1}\right)$ of length $2 g-2 h-1$. Put

$$
\bar{C}_{2 g-2 h-1}:=\left(c_{2 h+3} c_{2 h+4} \cdots c_{2 g+1}\right)^{2 g-2 h} d_{h, 1}^{-1} d_{h, 2}^{-1} \in \mathcal{R} .
$$

We apply $\bar{C}_{2 g-2 h-1}^{-1}$-substitution to the right-hand side of the equivalence above and get a positive relator

$$
\begin{aligned}
\bar{F}_{g-h-1}^{\text {even }}:= & \left(c_{2} c_{3} \cdots c_{2 g+1}\right)^{2 g+2 h+2}\left(c_{2 g-2 h+1} \cdots c_{3} c_{2}\right)\left(c_{2 g-2 h+2} \cdots c_{4} c_{3}\right) \\
& \cdots\left(c_{2 g+1} \cdots c_{2 h+3} c_{2 h+2}\right) d_{h, 1} d_{h, 2} .
\end{aligned}
$$

Since $d_{h-1,1}, d_{h-1,2}, d_{h, 1}$ and $d_{h, 2}$ bound a subsurface of $\Sigma_{g}$ homeomorphic to $\Sigma_{0,4}$, we can draw simple closed curves $\bar{a}_{h}, \bar{b}_{h}$ and $\bar{c}_{h}$ on it as in $\S 3.2$ so that

$$
L_{h}:=\bar{a}_{h} \bar{b}_{h} \bar{c}_{h} d_{h-1,1}^{-1} d_{h-1,2}^{-1} d_{h, 1}^{-1} d_{h, 2}^{-1}
$$

is a lantern relator. Then we apply $L_{h}$-substitution to $\bar{F}_{g-h-1}^{\text {even }} F_{h-1}^{\text {even }}$ and obtain a positive relator

$$
\begin{aligned}
V_{h}:= & \left(c_{2} c_{3} \cdots c_{2 g+1}\right)^{2 g+2 h+2}\left(c_{2 g-2 h+1} \cdots c_{3} c_{2}\right)\left(c_{2 g-2 h+2} \cdots c_{4} c_{3}\right) \\
& \quad \cdots\left(c_{2 g+1} \cdots c_{2 h+3} c_{2 h+2}\right) \cdot \bar{a}_{h} \bar{b}_{h} \bar{c}_{h} \\
\cdot & \left(c_{2 h} c_{2 h-1} \cdots c_{2} c_{1}\right)\left(c_{2 h+1} c_{2 h} \cdots c_{3} c_{2}\right) \\
& \cdots\left(c_{2 g} c_{2 g-1} \cdots c_{2 g-2 h+2} c_{2 g-2 h+1}\right)\left(c_{1} c_{2} \cdots c_{2 g}\right)^{4 g-2 h+2}
\end{aligned}
$$

and the corresponding Lefschetz fibration $M_{h}^{V} \rightarrow S^{2}$ of genus $g$.

Signatures of $M^{U}$ and $M_{h}^{V}$ are computed from signatures of chain and lantern relators by virtue of Theorems 4.2 and 4.3 :

$$
\begin{aligned}
\operatorname{Sign}\left(M^{U}\right) & =I_{g}\left(C_{7}\right)+2 I_{g}(A)+I_{g}(L) \\
& =-31, \\
\operatorname{Sign}\left(M_{h}^{V}\right) & =2 I_{g}\left(C_{2 g}\right)+2 I_{g}(A)-I_{g}\left(C_{2 h-1}\right)-I_{g}\left(C_{2 g-2 h-1}\right)+I_{g}(L) \\
& =-6 g^{2}-8 g-4 g h+4 h^{2}-3 .
\end{aligned}
$$

The Euler characteristic and the slope of $M^{U}$ are 47 and 17/6, those of $M_{2}^{V}$ of $g=4$ are 255 and $31 / 10$, and those of $M_{2}^{V}$ of $g=5$ are 393 and $151 / 46$, respectively. Thus we have the next proposition.

Proposition 4.11. The Lefschetz fibration $M^{U} \rightarrow S^{2}$ of genus 3 and the Lefschetz fibration $M_{2}^{V} \rightarrow S^{2}$ of genus 4 and 5 are not isotopic to any holomorphic Lefschetz fibration over $\mathbb{C} P^{1}$.

4.5. Concluding remarks. We can generalize methods in $\S 4.3$ and $\S 4.4$. For example, it is possible to construct two or more "subchain parts" in a chain and apply substitutions twice or more. It is also possible to carry out the construction in $\S 4.4$ starting with two chains of length $2 g+1$ instead of two chains of length $2 g$, or with $n+1$ chains and $n$ lanterns instead of two chains and a lantern. As a result, we have more non-holomorphic Lefschetz fibrations of genus 3, 4 and 5 . 
Many of the known examples of non-holomorphic Lefschetz fibrations are "noncomplex" because they do not satisfy the Noether inequality though minimal or they cannot be found in the table of the Enriques-Kodaira classification of compact complex surfaces. For example, Fintushel and Stern [14] gave an example of minimal symplectic Lefschetz fibrations of genus $g$ with $K^{2}=g-2$ and $\chi_{h}=g+1$. A computation of the Seiberg-Witten invariants shows the minimality of their examples.

All examples exhibited in $\S 4$ satisfy the slope inequality $\lambda_{f} \geq 4-4 / g$ due to Xiao [45], but some of them violate lower bounds of the slope for non-hyperelliptic holomorphic fibrations of genus 3,4 and 5 . We began with a typical hyperelliptic Lefschetz fibration, which is on the slope bound $4-4 / g$, and then destroyed its hyperellipticity not to increase the slope so much. Thus we obtained examples of non-holomorphic Lefschetz fibration without proving their minimality. However, we could not achieve decreasing the slope of fibrations $M_{1}, M_{2}$ and $M_{3}$ by the substitution technique. All examples known to the authors have the slope greater than or equal to $4-4 / g$. For example, the above example due to Fintushel and Stern has the slope $9 / 2-5 / g$. We shall restate the following conjecture in terms of the slope.

Conjecture 4.12 (Hain, cf. [1], Question 5.10). The slope inequality $\lambda_{f} \geq 4-4 / g$ holds for every smooth Lefschetz fibration $f: M \rightarrow S^{2}$ of genus $g \geq 2$ over the 2-sphere.

We do not know if our examples having slope greater than lower bounds given by Konno 23], 24] and Chen 8] are holomorphic.

\section{Appendix A. Two Definitions of the Signature COCYCle}

We review two definitions of the signature cocycle due to Meyer [27] and Turaev [43] and prove that they coincide.

Meyer [27] discovered the signature cocycle and gave an explicit description as follows.

Definition A.1 (Meyer [27]). Let $A, B \in S p(2 g, \mathbb{Z})$ be symplectic matrices of rank $2 g$. We consider the subspace

$$
V_{A, B}:=\left\{(x, y) \in \mathbb{R}^{2 g} \times \mathbb{R}^{2 g} \mid\left(A^{-1}-I_{2 g}\right) x+\left(B-I_{2 g}\right) y=0\right\}
$$

of the real vector space $\mathbb{R}^{2 g} \times \mathbb{R}^{2 g}$, where $I_{2 g}$ is the identity matrix. The bilinear form $\langle\quad, \quad\rangle_{A, B}:\left(\mathbb{R}^{2 g} \times \mathbb{R}^{2 g}\right) \times\left(\mathbb{R}^{2 g} \times \mathbb{R}^{2 g}\right) \rightarrow \mathbb{R}$ is defined by

$$
\left\langle\left(x_{1}, y_{1}\right),\left(x_{2}, y_{2}\right)\right\rangle_{A, B}:=\left(x_{1}+y_{1}\right) \cdot J\left(I_{2 g}-B\right) y_{2},
$$

where - is the inner product of $\mathbb{R}^{2 g}$ and $J$ is the matrix representing the multiplication by $\sqrt{-1}$ on $\mathbb{R}^{2 g}=\mathbb{C}^{g}$. It is easy to see that the restriction of $\langle,\rangle_{A, B}$ on $V_{A, B}$ is symmetric. Then we define

$$
\tau_{g}(A, B):=\operatorname{sign}\left(V_{A, B},\langle\quad, \quad\rangle_{A, B}\right) .
$$

$\tau_{g}$ turns out to be a 2-cocycle of $S p(2 g, \mathbb{Z})$ and called the signature cocycle of genus $g$.

Turaev [43] rediscovered (independently) the cocycle above. 
Definition A.2 (Turaev [43]). Let $A, B \in S p(2 g, \mathbb{Z})$ be symplectic matrices of rank $2 g$. We consider the subspace

$$
W_{A, B}:=\left(A-I_{2 g}\right) \mathbb{R}^{2 g} \cap\left(B-I_{2 g}\right) \mathbb{R}^{2 g}
$$

of the real vector space $\mathbb{R}^{2 g}$. The bilinear form ( , $)_{A, B}: W_{A, B} \times W_{A, B} \rightarrow \mathbb{R}$ is defined by

$$
\left(\xi_{1}, \xi_{2}\right)_{A, B}:=\left(u+v+\xi_{1}\right) \cdot J \xi_{2},
$$

where $\xi_{1}, \xi_{2} \in W_{A, B}$ and $u, v \in \mathbb{R}^{2 g}$ such that $\xi_{1}=A u-u=B v-v$. It is easy to see that $(, \quad)_{A, B}$ is well defined and symmetric. We define

$$
\varphi_{g}(A, B):=\operatorname{sign}\left(W_{A, B},(\quad, \quad)_{A, B}\right) .
$$

$\varphi_{g}$ turns out to be a 2 -cocycle of $S p(2 g, \mathbb{Z})$.

For $A, B \in S p(2 g, \mathbb{Z})$, we define the linear map $f_{A, B}: V_{A, B} \rightarrow W_{A, B}$ by

$$
f_{A, B}(x, y):=(B-I) y \quad\left((x, y) \in V_{A, B}\right) .
$$

It is easy to check that this map is well defined.

Proposition A.3. For $A, B \in S p(2 g, \mathbb{Z})$, the linear map $f_{A, B}: V_{A, B} \rightarrow W_{A, B}$ is surjective and satisfies

$$
\left(f_{A, B}(x, y), f_{A, B}\left(x^{\prime}, y^{\prime}\right)\right)_{A, B}=-\left\langle(x, y),\left(x^{\prime}, y^{\prime}\right)\right\rangle_{A, B},
$$

where $(x, y),\left(x^{\prime}, y^{\prime}\right) \in V_{A, B}$. In particular, $\varphi_{g}(A, B)=-\tau_{g}(A, B)$.

Proof. Let $\xi$ be an element of $W_{A, B}$. We take $u, v \in \mathbb{R}^{2 g}$ so that $\xi=A u-u=B v-v$. This equality means

$$
\left(A^{-1}-I\right)(A u)+(B-I) v=0
$$

and implies $(A u, u) \in V_{A, B}$. So we have $f_{A, B}(A u, u)=(B-I) v=\xi$.

For $(x, y),\left(x^{\prime}, y^{\prime}\right) \in V_{A, B}$, we have

$$
\begin{aligned}
& \left(f_{A, B}(x, y), f_{A, B}\left(x^{\prime}, y^{\prime}\right)\right)_{A, B}=\left((B-I) y,(B-I) y^{\prime}\right)_{A, B} \\
= & \left((A-I)^{-1}((B-I) y)+(B-I)^{-1}((B-I) y)+(B-I) y\right) \cdot J(B-I) y^{\prime} \\
= & \left((A-I)^{-1}\left((A-I)\left(A^{-1} x\right)\right)+y+B y-y\right) \cdot J(B-I) y^{\prime} \\
= & \left(A^{-1} x+B y\right) \cdot J(B-I) y^{\prime}=-\left(A^{-1} x+B y\right) \cdot(I-B) y^{\prime} \\
= & -(x+y) \cdot(I-B) y^{\prime} \\
= & -\left\langle(x, y),\left(x^{\prime}, y^{\prime}\right)\right\rangle_{A, B} .
\end{aligned}
$$

Because the kernel of $f_{A, B}$ is included in the annihilator of $\langle\quad, \quad\rangle_{A, B}, f_{A, B}$ induces a linear isomorphism

$$
\bar{f}_{A, B}:\left(V_{A, B} / \operatorname{Ker} f_{A, B},-\langle\quad, \quad\rangle_{A, B}\right) \rightarrow\left(W_{A, B},(\quad, \quad)_{A, B}\right)
$$

which preserves bilinear forms.

The mapping class group $\mathcal{M}_{g}$ of genus $g$ acts on $H_{1}\left(\Sigma_{g} ; \mathbb{Z}\right)$ preserving the intersection form. If we fix a symplectic basis on $H_{1}\left(\Sigma_{g} ; \mathbb{Z}\right)$, we obtain a representation $\mathcal{M}_{g} \rightarrow S p(2 g, \mathbb{Z})$. We call the pull-back of the signature cocycle by this representation also the signature cocycle.

Remark A.4. The signature cocycle satisfies the condition (*) in Proposition 2.3 (see Meyer [27] formula (9)). 


\section{REFERENCES}

[1] J. Amorós, F. Bogomolov, L. Katzarkov and T. Pantev, Symplectic Lefschetz fibrations with arbitrary fundamental groups, J. Diff. Geom. 54 (2000), 489-545. MR 2002g:57051

[2] T. Arakawa and T. Ashikaga, Local splitting families of hyperelliptic pencils, I, Tohoku Math. J. 53 (2001), 369-394. MR 2002h:14041

[3] T. Ashikaga and K. Konno, Global and local properties of pencils of algebraic curves, Algebraic Geometry 2000, Azumino, Advanced Studies in Pure Mathematics 36, 2000, pp. 1-49. MR 2003k:14003

[4] D. Auroux, Fiber sums of genus 2 Lefschetz fibrations, Turkish J. Math. 25 (2001), 1-10. MR 2004b:57033

[5] J. Birman and H. Hilden, On mapping class groups of closed surfaces as covering spaces, Advances in the Theory of Riemann surfaces, Ann. Math. Stud. 66, Princeton Univ. Press, 1971, pp. 81-115. MR 45:1169

[6] K. S. Brown, Cohomology of Groups, Graduate Texts in Mathematics 87, Springer-Verlag, 1982. MR $83 \mathrm{k}: 20002$

[7] C. Cadavid, A remarkable set of words in the mapping class group, Dissertation, Univ. of Texas, Austin, 1998.

[8] Z. Chen, On the lower bound of the slope of a non-hyperelliptic fibration of genus 4, Intern. J. Math. 4 (1993), 367-378. MR 94g:14012

[9] M. Dehn, Die Gruppe der Abbildungsklassen, Acta Math. 69 (1938), 135-206.

[10] S. K. Donaldson, Lefschetz pencils on symplectic manifolds, J. Diff. Geom. 53 (1999), 205236. MR 2002g:53154

[11] C. J. Earle and J. Eells, A fibre bundle description of Teichmüller theory, J. Diff. Geom. 3 (1969), 19-43. MR 43:2737a

[12] H. Endo, Meyer's signature cocycle and hyperelliptic fibrations (with Appendix written by T. Terasoma), Math. Ann. 316 (2000), 237-257. MR 2001b:57047

[13] H. Endo, M. Korkmaz, D. Kotschick, B. Ozbagci and A. Stipsicz, Commutators, Lefschetz fibrations and the signatures of surface bundles, Topology 41 (2002), 961-977. MR 2003f:57051

[14] R. Fintushel and R. J. Stern, Constructions of smooth 4-manifolds, Documenta Mathematica, Extra Volume ICM 1998, II, pp. 443-452. MR 99g:57033

[15] T. Fuller, Diffeomorphism types of genus 2 Lefschetz fibrations, Math. Ann. 311 (1998), 163-176. MR 99f:57035

[16] S. Gervais, Presentation and central extensions of mapping class groups, Trans. Amer. Math. Soc. 348 (1996), 3097-3132. MR 96j:57016

[17] R. E. Gompf, A topological characterization of symplectic manifolds, preprint, math.SG /0210103.

[18] R. E. Gompf and A. I. Stipsicz, 4-manifolds and Kirby calculus, Graduate Studies in Mathematics 20, American Mathematical Society, 1999. MR 2000h:57038

[19] H. Hopf, Fundamentalgruppe und zweite Bettische Gruppe, Comment. Math. Helv. 14 (1942), 257-309. MR 3:316e

[20] T. Ito, Splitting of singular fibers in certain holomorphic fibrations, J. Math. Sci. Univ. Tokyo 9 (2002), 425-480. MR 2003h:32040

[21] D. Johnson, The structure of the Torelli group I: A finite set of generators for $\mathcal{I}$, Ann. of Math. 118 (1983), 423-442. MR 85a:57005

[22] A. Kas, On the handlebody decomposition associated to a Lefschetz fibration, Pacific J. Math. 89 (1980), 89-104. MR 82f:57012

[23] K. Konno, A note on surfaces with pencils of non-hyperelliptic curves of genus 3, Osaka J. Math. 28 (1991), 737-745. MR 93c:14035

[24] K. Konno, Non-hyperelliptic fibrations of small genus and certain irregular canonical surfaces, Ann. Sc. Norm. Pisa Sup. Ser.IV, vol.XX (1993), 575-595. MR 95b:14026

[25] M. Korkmaz, Noncomplex smooth 4-manifolds with Lefschetz fibrations, Internat. Math. Res. Not. 2001, No. 3, 115-128. MR 2001m:57036

[26] F. Luo, A presentation of mapping class groups, Math. Res. Lett. 4 (1997), 735-739. MR 99b:57031

[27] W. Meyer. Die Signatur von Flächenbündeln, Math. Ann. 201 (1973), 239-264. MR 48:9715

[28] Y. Matsumoto, On 4-manifolds fibered by tori, II, Proc. Japan Acad. 59 (1983), 100-103. MR $84 \mathrm{j}: 57010 \mathrm{~b}$ 
[29] Y. Matsumoto, Lefschetz fibrations of genus two - a topological approach -, Proceedings of the 37th Taniguchi Symposium on "Topology and Teichmüller Spaces", World Scientific, Singapore, 1996, pp. 123-148. MR 2000h:14038

[30] T. Morifuji, On Meyer's function of hyperelliptic mapping class groups, J. Math. Soc. Japan 55 (2003), 117-129. MR 2003m:57054

[31] S. Morita, Characteristic classes of surface bundles, Invent. Math. 90 (1987), 551-577. MR 89e:57022

[32] S. Nagami, A note on orientations of fixed point sets of spin structure preserving involutions, to appear in Kobe J. Math.

[33] B. Ozbagci, Signatures of Lefschetz fibrations, Pacific J. Math. 202 (2002), 99-118. MR 2002k:57066

[34] B. Ozbagci and A. Stipsicz, Noncomplex smooth 4-manifolds with genus-2 Lefschetz fibrations, Proc. Amer. Math. Soc. 128 (2000), 3125-3128. MR 2000m:57036

[35] B. Siebert and G. Tian, On hyperelliptic $C^{\infty}$-Lefschetz fibrations of four-manifolds, Commun. Contemp. Math. 1 (1999), 466-488. MR 2001g:57053

[36] I. Smith, Lefschetz fibrations and the Hodge bundle, Geometry \& Topology 3 (1999), 211-233. MR 2000j:57059

[37] I. Smith, Lefschetz pencils and divisors in moduli space, Geometry \& Topology 5 (2001), 579-608. MR 2002f:57056

[38] A. I. Stipsicz, On the number of vanishing cycles in Lefschetz fibrations, Math. Res. Lett. 6 (1999), 449-456. MR 2000g:57046

[39] A. I. Stipsicz, Indecomposability of certain Lefschetz fibrations, Proc. Amer. Math. Soc. 129 (2000), 1499-1502. MR 2001h:57029

[40] A. I. Stipsicz, Spin structures on Lefschetz fibrations, Bull. London Math. Soc. 33 (2001), 466-472. MR 2002a:53062

[41] A. I. Stipsicz, Singular fibers in Lefschetz fibrations on manifolds with $b_{2}^{+}=1$, Topology and its Appl. 117 (2002), 9-21. MR 2002j:57048

[42] T. Terasoma, An appendix to Endo's paper, Math. Ann. 316 (2000), 255-256. MR 2001b:57047

[43] V. G. Turaev, First symplectic Chern class and Maslov indices, J. Soviet Math. 37 (1987), 1115-1127. MR 86m:58059

[44] B. Wajnryb, An elementary approach to the mapping class group of a surface, Geometry \& Topology 3 (1999), 405-466. MR 2001a:20059

[45] G. Xiao, Fibered algebraic surfaces with low slope, Math. Ann. 276 (1987), 449-466. MR 88a:14046

Department of Mathematics, Graduate School of Science, Osaka University, ToyONAKA, OSAKA 560-0043, JAPAN

E-mail address: endo@math.wani.osaka-u.ac.jp

3-6-3-10 Sakuranchou, Toyonaka, Osaka 560-0054, Japan

E-mail address: nagami-s@est.hi-ho.ne.jp 